\title{
Pencil beam characteristics of the next-generation proton scanning gantry of PSI: design issues and initial commissioning results
}

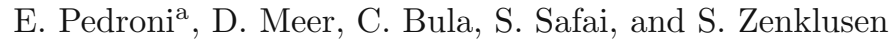 \\ Center for Proton Therapy, Paul Scherrer Institute - CH 5232 Villigen-PSI, Switzerland
}

Received: 28 April 2011

Published online: 25 July 2011 - (C) Società Italiana di Fisica / Springer-Verlag 2011

\begin{abstract}
In this paper we report on the main design features, on the realization process and on selected first results of the initial commissioning of the new Gantry 2 of PSI for the delivery of proton therapy with new advanced pencil beam scanning techniques. We present briefly the characteristics of the new gantry system with main emphasis on the beam optics, on the characterization of the pencil beam used for scanning and on the performance of the scanning system. The idea is to give an overview of the major components of the whole system. The main long-term technical goal of the new equipment of Gantry 2 is to expand the use of pencil beam scanning to the whole spectrum of clinical indications including moving targets. We report here on the initial experience and problems encountered in the development of the system with selected preliminary results of the ongoing commissioning of Gantry 2.
\end{abstract}

\section{Introduction}

Proton therapy with pencil beam scanning has been pioneered by PSI in the 90's, by developing the first scanning gantry in the world, the Gantry 1 which has been used routinely for patient treatments since 1996 at this institute. A major limitation of this system is the sensitivity of pencil beam scanning to organ motion during beam delivery: With Gantry 1 we can treat only non-moving targets due to possible interferences of the target motion with the dynamic motion of the pencil beam, which can produce substantial errors in the homogeneity of the dose distribution within the target [1].

In order to overcome this difficulty a new gantry, Gantry 2 has been built at PSI. The new system was conceived for the development of much faster scanning techniques, in order to provide the capability to re-irradiate the target multiple times (volumetric repainting). On Gantry 2 the dose is delivered with a fast double parallel scanning and by changing the energy with the beam line very quickly. "Dose energy-layers" are painted to the shape of the target with non-homogeneous proton fluence such as to deliver a conformal dose distribution to the target without needing patient's or field's specific hardware. Multiple energy layers are combined with fast energy changes in the beam line to create a well-optimized 3d-dose distribution delivered with precision close to the physical limit. For best precision the scan must be performed with a well focused small pencil beam. The requirements to achieve a rather high dose rate while scanning the beam at high scanning speed (in position and range) using a small sharp pencil beam, were the basic optimization criteria for designing the new gantry system.

A major intention of the paper is to show the importance of the role played by particle physics and accelerator physics towards the goal of designing an excellent radiation therapy instrument.

The new Gantry 2 has been completed by the end of 2010. The system is under commissioning now. In this report we describe the basic characteristics of the system and we report on the initial experience of realizing the new gantry and present preliminary results on the characteristics of the pencil beam and on the achieved speed of scanning.

\section{The development framework: the PSI proton therapy facility and its history}

PSI has a long tradition in using particle beams for cancer treatments.

\footnotetext{
${ }^{a}$ e-mail: eros.pedroni@psi.ch
} 


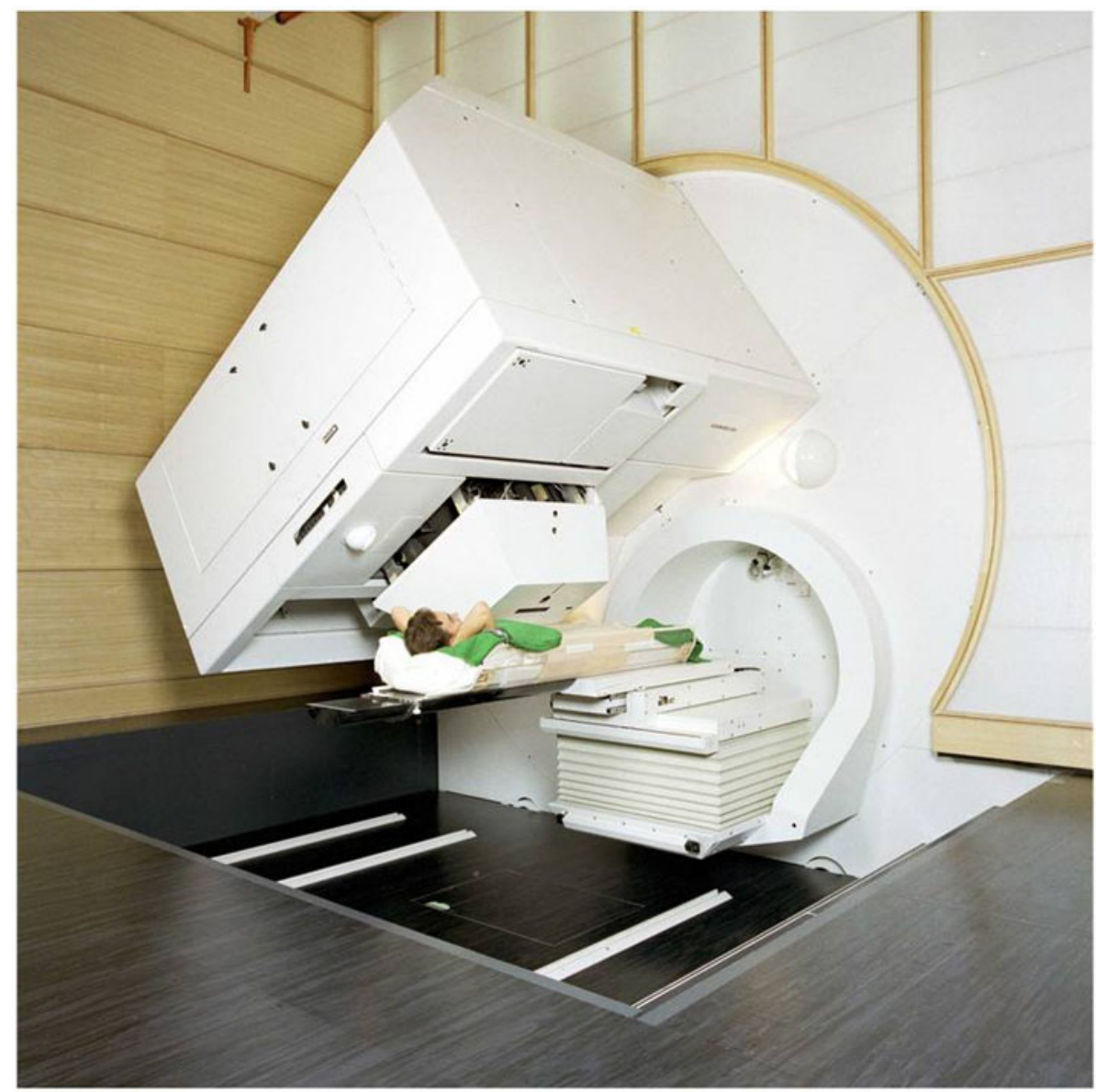

Fig. 1. Photograph of Gantry 1, the system used since 1996 for treating patients with pencil beam scanning at PSI.

From 1981 until 1992 pion therapy was used for treating more than 500 patients with a very advanced scanning technology [2] but unfortunately with the "wrong type of particle", the $\pi^{-}$mesons, which are better replaced today by light ions. Pion therapy was abandoned in 1992 in favor of the realization of the first scanning gantry for proton therapy (the Gantry 1 system described below).

Uveal melanomas (tumors in the interior of the eye) are being treated at PSI since 1984. This therapy has been applied with the $72 \mathrm{MeV}$ beam of the Injector 1 of PSI in the facility Optis 1 until 2010, when the old area was replaced by a new one, Optis 2, integrated in the new proton therapy complex of PSI. The transfer was accomplished in the context of the expansion of the whole facility, the so-called PROSCAN project described below. More than 5500 patients, the largest amount in a facility worldwide, have been treated at PSI with protons for this indication [3].

The decision to build a first gantry [4] dedicated to pencil beam scanning with protons was taken in 1991. Since 1996 patients are being treated with pencil beam scanning on this first gantry system (Gantry 1). The Gantry 1 of PSI has been for over a decade the only proton gantry system in the world treating patients with pencil beam scanning. A similar scanning facility has been operational with carbon ions in a horizontal beam line at GSI in Germany [5]. Only in 2008 a few commercial proton gantries (in Houston and Boston) started operation with the pencil beam scanning technique, as an optional alternative to the more established passive scattering technique. Meanwhile many new commercial systems are being or have been built based completely on scanning (Munich, Essen and Trento). This evolution is in part due to the pioneering work of PSI in developing the technology of pencil beam scanning with protons on a gantry.

Figure 1 shows the treatment area of Gantry 1. With a $2 \mathrm{~m}$ radius this system is still the most compact proton gantry in the world.

With Gantry 1 we could show the potential advantages of scanning and we have also pioneered the development of intensity modulated proton therapy (IMPT) [6]. The possibility to simultaneously optimize fields while using multiple beam directions was already realized within the pion therapy approach $[7,8]$. IMPT is considered today a new necessary proton technique for competing with IMRT in conventional therapy. IMPT is presently used at PSI for about $1 / 3$ of the patients.

The various advantages of using scanning have been discussed in other reports (see for example [9]). 


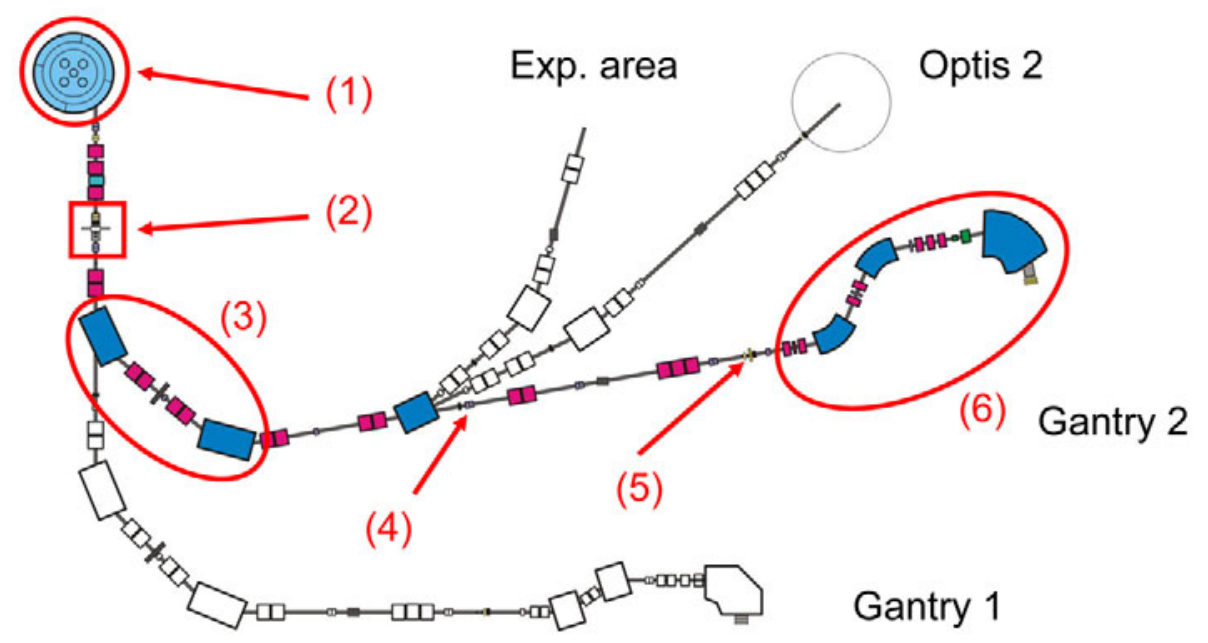

Fig. 2. The layout of the proton therapy facility at PSI. Highlighted is the beam line to the Gantry 2. The system comprises the cyclotron COMET (1), the degrader (2), the analyzing section (3), intensity suppression collimator (4) (see also sect. 8.2.1), coupling point (5) to the gantry and Gantry 2 (6).

Gantry 1 has been used for most of its lifetime in parasitic mode to physics experiments in a complex research environment. The Gantry 1 beam was obtained until 2006 by splitting a small fraction ( $0.5 \%$ of the beam intensity) of the beam of the PSI's ring cyclotron and by degrading the energy of the beam from $590 \mathrm{MeV}$ down to the required energy in the range between $120 \mathrm{MeV}$ and $214 \mathrm{MeV}$. Despite these logistic difficulties the project has been in the end quite successful.

This success brought in 2000 the decision to expand the proton facility at PSI and to launch the so-called project PROSCAN.

In 2000 the PSI directorate decided in favor of purchasing a new $250 \mathrm{MeV}$ superconducting cyclotron dedicated to the proton therapy project. The new accelerator (designed by Henry Blosser at the Michigan State University in the USA) was delivered by industry (ACCEL GmbH in Germany).

Figure 2 shows the layout of the expanded proton facility of PSI. Three new areas, Optis 2, Gantry 2 and PIF (an experimental area for physics experiments) have been added to the existing Gantry 1 area. The facility (excluding the cyclotron) has been realized internally by the technical divisions of PSI.

The degrader and the beam switchyard were realized under the condition to maintain the Gantry 1 treatments uninterrupted as long as possible using the old connection to the ring cyclotron while installing the rest of the new facility [10]. The beam from the new accelerator was switched to the Gantry 1 at the last moment. This development history is reflected in the unusual shape of the facility layout.

Gantry 1 is in use for patient treatments with the new dedicated accelerator COMET since February 2007. Since summer 2007 patients are treated at Gantry 1 all the year through without planned shut-downs of more than three days.

A new area of the PROSCAN facility is dedicated to the realization of a next-generation scanning gantry, Gantry 2, a new improved system based on the experience gained with Gantry 1. For Gantry 2 we plan to increase considerably the speed of scanning in order to be able to paint the dose repeatedly and to be able to treat moving tumors with repainting and gating. Another possibility under discussion is the use of a breath-hold technique for treating lung tumors.

The specifications for the whole project PROSCAN were defined in view of the development of the new advanced beam scanning techniques for Gantry 2 [11].

\section{The mechanical layout of Gantry 2}

In this section we describe the mechanical characteristics of the new Gantry 2.

At the time of writing the Gantry 2 looks as shown in fig. 3. We could send the first beam through the gantry in 2008 but due to limited resources the other installations in the gantry area were completed only in 2010 . The initial ideas for the system were presented in a first publication in 2005 [11]. Some important changes were applied during the realization of the system, the most noticeable difference to [11] being the way of supporting the gantry, which is now by a central commercial pivot bearing before the last bending magnet. This improves the access to the iso-center, since the area is open from the front and from the side of the gantry. 


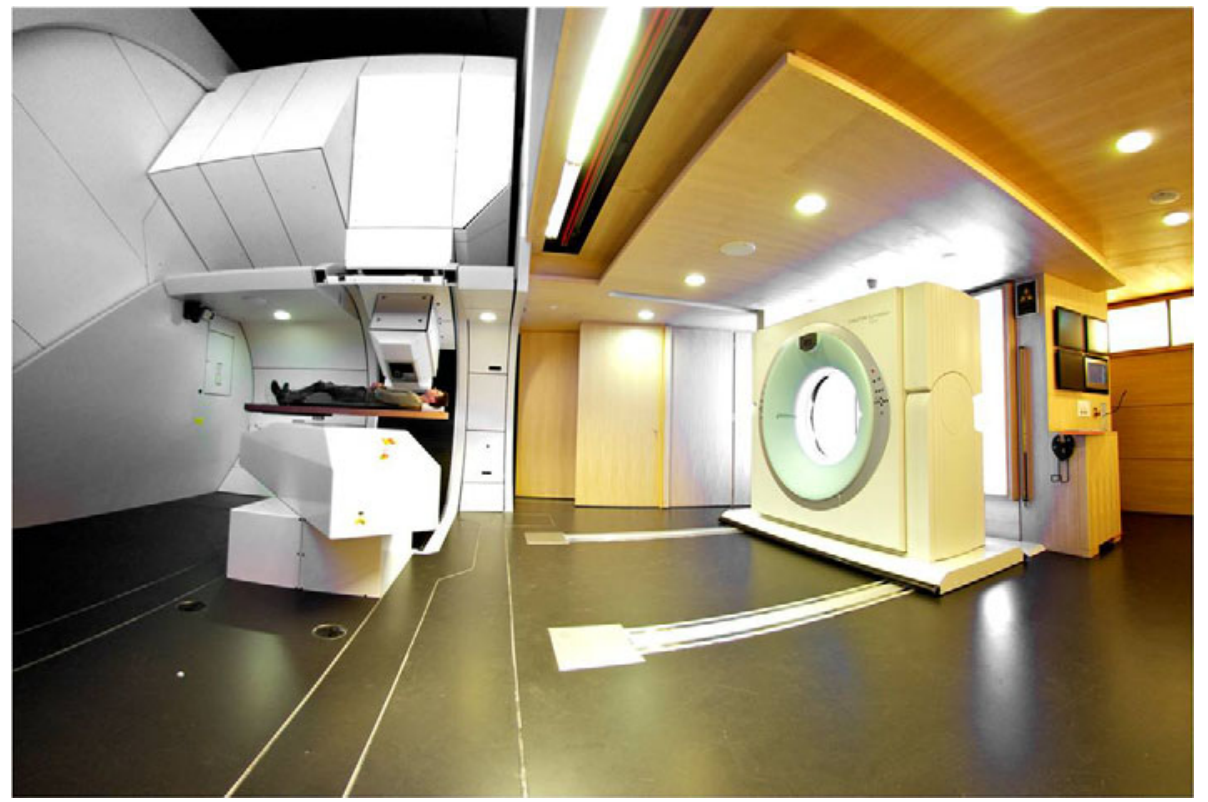

Fig. 3. The layout of the Gantry 2 room with patient table, nozzle and the sliding CT (a fish eye photograph).

The overall layout of the rotating beam line is shown in fig. 4 (picture generated as a CAD picture of the beam line and of the rotating mechanical support).

The parallel displacement of the beam axis from the central rotation axis of the gantry was chosen at a distance of $3.2 \mathrm{~m}$. The diameter spanned by the mechanical system of the new iso-centric Gantry 2 is about $8.4 \mathrm{~m}$. The distance of the beam exit (the pole edge) of the last $90^{\circ}$ bending magnet to the iso-center is $1.7 \mathrm{~m}$, which is considerably larger than the value of $1.1 \mathrm{~m}$ used for Gantry 1 . This extra space was assigned for keeping the rotation of the $90^{\circ}$ bending magnet outside of the half-cylinder cell of the treatment area (see fig. 3) with a distance from false floor to false ceiling of about $2.3 \mathrm{~m}$, a value similar to a usual room.

The mechanical systems (gantry support and patient table) have been delivered by the Swiss company Schär Engineering Ltd. The other gantry components have been realized internally at PSI.

In the remaining of this section we describe the mechanical characteristics of the new Gantry 2.

\subsection{Iso-centric layout}

As opposed to Gantry 1, which is very compact but eccentric (patient table mounted on the gantry and rotating with the gantry), Gantry 2 has been built as an iso-centric system. The easy access to the patient table at any time on a fixed floor was judged to be the most important characteristic of the new Gantry 2 system.

\subsection{Gantry rotation limited to $-30^{\circ}$ to $+180^{\circ}$ (one-sided rotation)}

An unusual feature of the system is the limited rotation of the gantry, which is from $-30^{\circ}$ to $+180^{\circ}$. The gantry thus rotates only on one side. The patient table is mounted at the opposed side (i.e. along the beam axis when the gantry is horizontal at a gantry angle of $+90^{\circ}$ ).

The original goal was to design a treatment room, which looks like a usual square room with the exception of the half-cylindrical wall where the nozzle rotates from $0^{\circ}$ to $180^{\circ}$ in order to not see anything of the gantry except the nozzle. Later we decided to extend the rotation to $-30^{\circ}$. This should make the system quicker in the choice of the treatment angles when treating with beam from above, but at the price of a mechanically more complex and costly system.

The flexibility to choose any desired beam incidence is achieved by rotating the patient table in the horizontal plane. In the new gantry layout, we attempted to combine the advantages of a horizontal line with the flexibility of a gantry.

\section{Gantry room with a permanent fixed floor}

By not having a full rotation of the last $90^{\circ}$ bending magnet, we could avoid the usual problems of having an open gantry pit in the area at the iso-center. We can provide a fixed "false floor" in the treatment room, which is supported from the outside of the gantry pit ands is not "cut" by the rotation of the gantry head. 


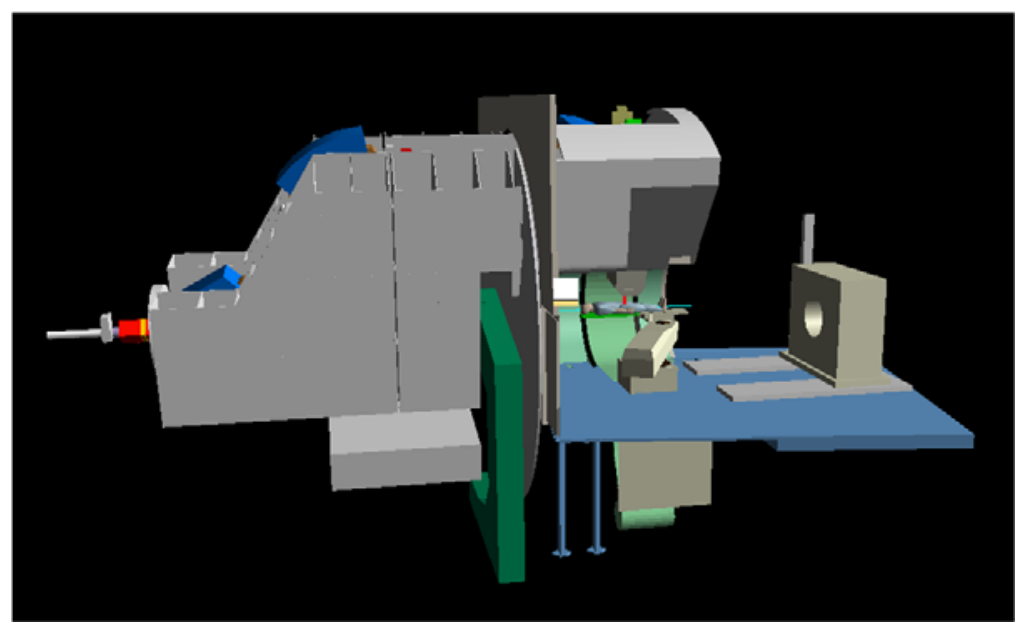

Fig. 4. Mechanical layout of the gantry with rotating support (gray), U-profile with pivot bearing (green), gantry head with the $90^{\circ}$ bending magnet, patient table and sliding CT.

In this way we can provide an easy permanent access to the patient table at any time with exception of the slit region where the nozzle rotates, which is covered with a rolling floor.

We have also fixed walls and a (partial) fixed ceiling in the vicinity of the iso-center. This will permit to mount commercial diagnostic equipment in a fixed environment at the iso-center, e.g. planar lasers for marking the iso-center, devices for stereo viewing of the patient surfaces, etc.

\section{Gantry support with a commercial gantry bearing}

The limited rotation of the gantry allows supporting the gantry in the central region with a commercial pivot bearing. By this we avoid the use of an $8 \mathrm{~m}$ large cylinder as gantry support, which is difficult to fabricate to the required precision.

\section{Gantry with upstream scanning}

The sweeper magnets are mounted on the gantry before the last bending magnet. The scanning is started before bending the beam in the direction of the patient (the same solution as with Gantry 1 but with two sweeper magnets instead of only one). In this way we spare space in the beam line section after the last bending magnet, namely the space which would otherwise be occupied by the sweeper magnets and the drift space for the lateral spreading of the beam. This significantly reduces the overall radius of the gantry.

By using a solution with upstream scanning we can provide a parallel beam scanning in both lateral scanning directions as explained later in the beam optics section.

The upstream scanning solution is also best suited for the use of the BEV X-ray concept described below.

\subsection{Equipment for patient positioning and for image guided proton therapy}

\section{Patient table}

The patient table is of the same type as delivered by Schär Engineering Ltd. to the Rinecker Proton Therapy Center in Munich. The table is set on a vertical carriage at a distance of about $1.8 \mathrm{~m}$ from the iso-center. A first translational axis $Y$ moves the whole patient table vertically up and down over $70 \mathrm{~cm}$. A pivotal axis $\Phi$ on top of the vertical sledge provides the main central rotation of the upper part of the table. On top of the rotation $\Phi$-axis a radial support moves over a length of $2.6 \mathrm{~m}$ providing the second translation axis $L$. A second rotational axis $\Omega$ which can rotate by $\pm 141^{\circ}$ is mounted at the end of the radial support. On top of the $\Omega$-axis there is the clamping mechanism for mounting the actual patient support (the couch), which can be of different types and size and can be exchanged. The individual patient moulds are clamped on the couch support. The patient will be treated in supine position (see fig. 4).

Through the combination of the two rotations and one longitudinal translation of the patient table in the horizontal plane, it is possible to treat the patient in supine position with the tumor at the iso-center for all horizontal beam angles in a range of about $\pm 100^{\circ}-120^{\circ}$ with respect to the main patient body axis. Including the gantry rotation the degrees of freedom of choosing the beam incidence on the supine patient cover a solid angle of more than $2 \pi$. The beam incidences of interest are those with an entrance point in the patient's body sufficiently near in proton range to the tumor location. It does for example not make sense to treat a tumor in the patient head with beam through the feet. If we want to treat a tumor near the feet (from the feet) we should better tilt the patient position on the 


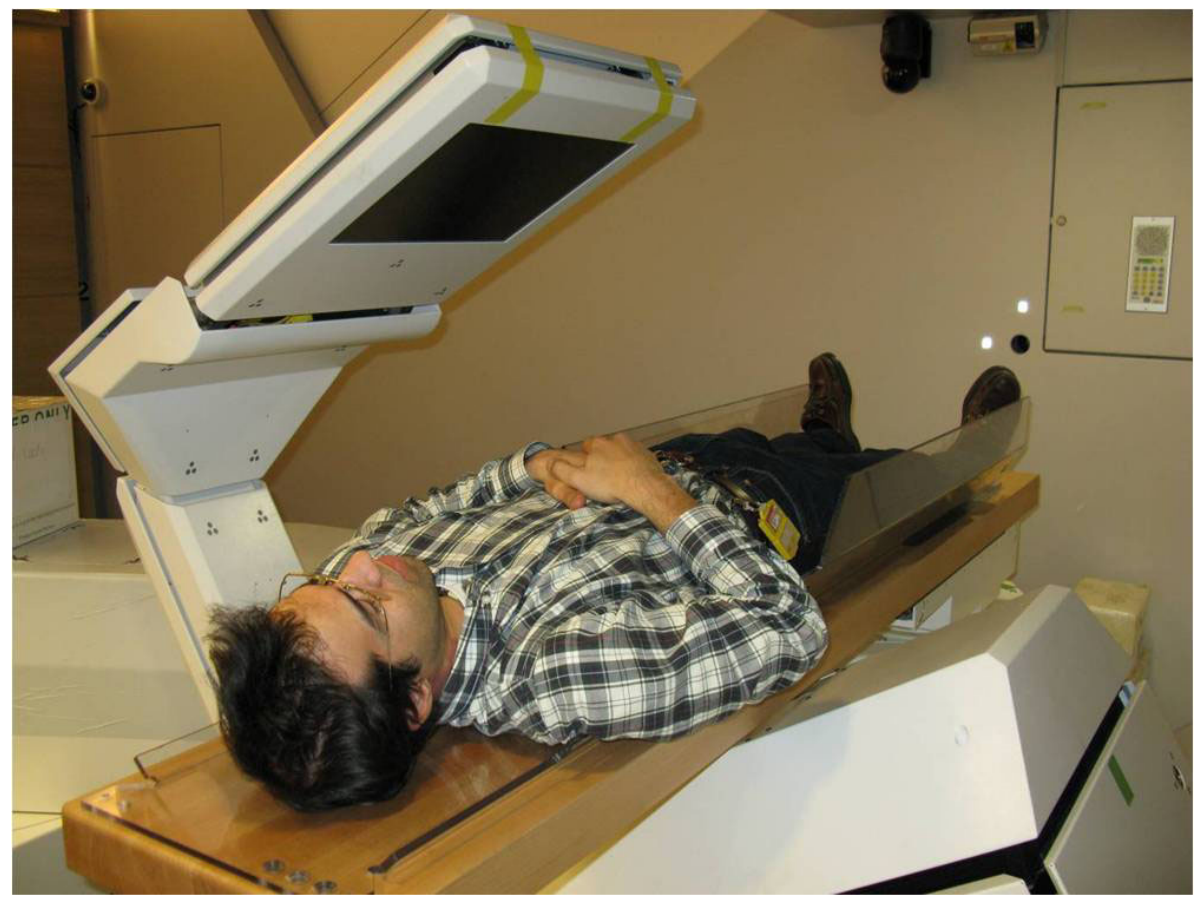

Fig. 5. Photograph of the retractable arm for holding the X-ray panel behind the patient on the side opposite to the nozzle (BEV X-ray).

couch. We are therefore convinced that our choice covers in practice all relevant situations quite similarly as with an idealized $4 \pi$ solution (which is in practice not feasible without compromises).

The patient table offers the possibility to apply pitch and roll corrections to the patient couch. This feature will not be supported in the first software release of the system for controlling the motion of the patient table. This option will probably be realized at a later time.

A major requirement for the mechanical systems has been to be able to move the gantry and the patient table under remote control. This brings the freedom to deliver dose fields sequentially without the need for the personnel to enter the room in between fields (the remote control of the patient table is at PSI a routine solution already for Gantry 1). As a consequence we must guarantee by automation for the patient's safety against possible mechanical collisions. This has been realized by dedicated safety hardware with collision detection devices and by software with "virtual walls" limiting the regions of the possible movements of the table. The software for the control of the mechanical devices for the positioning of the patient table and the gantry is being developed internally by PSI engineers.

\section{Sliding $C T$}

The layout of the gantry was conceived for maximum flexibility, permitting the installation of a sliding CT. The idea is to be able to install in the treatment area the best commercial diagnostic equipment available on the market, to be used within reach of the patient table, to check with highest precision the setup of the patient before and after the beam delivery. The possibility to take time-resolved images is of particular interest for treating tumors in the context of moving organs, for developing new quality assurance methods and for supporting the patient setup for gating, tracking or breath-hold techniques.

\section{$B E V$ X-ray}

The last $90^{\circ}$ bending magnet has been designed with a hole in the external return yoke, which allows shining X-ray through the last $90^{\circ}$ magnet along the nozzle.

On the gantry we have mounted a retractable support, which allows positioning an X-ray panel behind the patient for taking X-ray and fluoroscopy images.

Figure 5 shows a photograph of the holder of the X-ray panel.

The system will be used for sporadic checks of the position of the patient at the iso-center as a redundant check to the use of the sliding CT, following the same strategy established on Gantry 1.

In the long range we will explore the possibility to use the BEV X-ray system in synchronization (or simultaneously) with the proton beam delivery, provided that the neutrons arising from protons stopping in the patient will not damage 


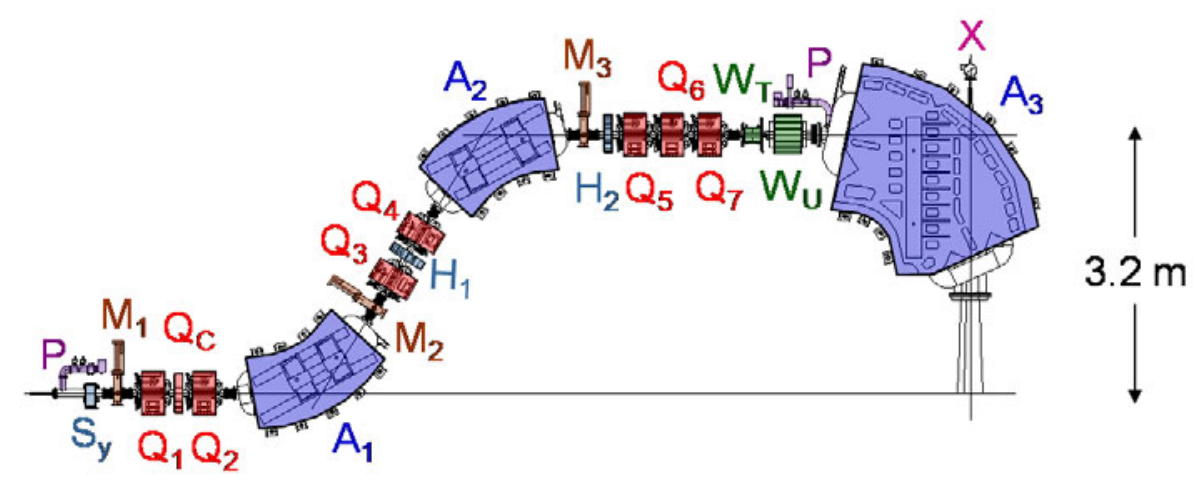

Fig. 6. The elements of the Gantry 2 beam line. Elements $A_{1-3}$ are dipoles, $Q_{1-7, C}$ quadrupoles, $W_{T}, W_{U}$ sweeper magnets used for scanning, $\mathrm{M}_{1-3}$ profile monitors, $\mathrm{P}$ vacuum pumps, $\mathrm{S}_{y}$ steering magnet, $\mathrm{H}_{1,2}$ sextupoles. $\mathrm{X}$ is the location of the $\mathrm{X}$-ray tube.

too quickly the panel. If it worked, this approach could open new prospective solutions for quality control and for proton beam guidance for treating moving targets with scanning beams.

More details on the solutions for the patient's handling and positioning system will be published when the medical physics commissioning is completed.

\section{The beam line of Gantry 2}

Figure 6 shows the layout of the gantry beam line. The system consists of three dipoles (elements A) and seven quadrupoles (elements Q). The essential elements for scanning the beam are the sweeper magnets $W_{T}$ and $W_{U}$.

As can be seen the beam line is extremely compact. We believe that it is not possible to reduce further the dimensions of the gantry, unless one introduces some new technology or relaxes the requirements on the performance of the system.

All magnets were designed by the magnet group of PSI and fabricated by industry (Sigmaphi Ltd.). A major goal was to achieve very fast changes of the beam energy in the beam line (we were aiming at energy changes of the order of 100-150 ms per energy steps corresponding to $5 \mathrm{~mm}$ proton range in water, see sect. 8.4 later). All the beam line elements from the accelerator to the Gantry 2 area are therefore laminated magnets.

\subsection{Elements of the gantry beam line}

\section{The $58^{\circ}$ dipoles}

The beam is bent first by two counteracting bending magnets of $58^{\circ}$ to become displaced and parallel to the gantry rotation axis at a distance of $3.2 \mathrm{~m}$.

The bending radius of the trajectory in the dipoles was chosen to be $1.5 \mathrm{~m}$ (a value somewhat larger than the $1.27 \mathrm{~m}$ used on the PSI Gantry 1) for all dipoles including the last $90^{\circ}$ bending magnet in order to avoid saturation effects in the poles observed at the highest energies in our first system. The nominal field at maximum energy (230 MeV) is $1.55 \mathrm{~T}$, to be below saturation level. The gap for the $58^{\circ}$ dipoles was chosen to be $7 \mathrm{~cm}$.

\section{Quadrupoles}

The beam line contains seven quadrupoles (the first two doublets bring the beam through the gap of the first two dipoles and the last triplet adjusts the shape and size of the beam at the final focus). The radial opening of the quadrupoles was chosen with a radius of $4 \mathrm{~cm}$. This is related to the angular acceptance of the beam line of about $\pm 9 \mathrm{mrad}$. The effective length was designed for $30 \mathrm{~cm}$.

\section{Correction elements and beam diagnostic elements}

In the beam line we have steering magnets, sextupoles, beam diagnostics devices and vacuum pump systems. Beam steering effects are in part realized within the sextupoles whose poles are connected in pairs to three separate power supplies. These elements can act simultaneously as sextupoles and/or as steering magnets (the elements denoted in fig. 6 as $\mathrm{H}$ and $\mathrm{S}_{y}$ ). We have in addition three beam diagnostic elements $\mathrm{M}$, and two vacuum pumps $\mathrm{P}$.

\section{Sweeper magnets with quadrupole corrector}

The main dynamics elements for the delivery of pencil beam scanning are the two sweeper magnets $\mathrm{W}_{\mathrm{T}}$ and $\mathrm{W}_{\mathrm{U}}$, which are installed before the last $90^{\circ}$ bending magnet. 
We use two sweeper magnets in contrast to Gantry 1 where the beam is scanned magnetically only in one transverse direction, the one within the dispersive plane.

The $\mathrm{T}$ sweeper displaces the parallel beam in the transverse direction over $\pm 6 \mathrm{~cm}$.

The effective length of the T magnet is $20 \mathrm{~cm}$, the gap $6 \mathrm{~cm}$.

The sweeper $U$ displaces the beam in the dispersive plane over $\pm 10 \mathrm{~cm}$. The magnet has an effective length of $40 \mathrm{~cm}$ and a gap of $9 \mathrm{~cm}$.

In order to obtain good focusing properties while scanning the beam, the poles of the U sweeper magnets are tapered (with a field index of $7 \mathrm{~mm} / 10 \mathrm{~cm}$ ).

In between the first two quadrupoles $\mathrm{Q}_{1}$ and $\mathrm{Q}_{2}$ we have added a very thin quadrupole corrector $\mathrm{Q}_{\mathrm{C}}$, whose role is to maintain the beam focused at the iso-center independently of the scanning. The quadrupole corrector is connected in series with the U sweeper (see beam optics discussion below).

The vacuum chamber of the sweepers is made of a non-conducting material to avoid eddy currents.

The beam line is designed to provide parallel scanning in both sweeper directions with an apparent source at the infinity (see beam optics design below).

The beam optics were designed for scanning the center of the beam over a rectangular area of at least $20 \mathrm{~cm} \times 12 \mathrm{~cm}$. The monitor's area is $15 \mathrm{~cm} \times 25 \mathrm{~cm}$ for accommodating also the half width of the beam.

For treating tumors larger than the magnetic scan range we will combine the magnetic scanning of the beam with displacements of the patient table under remote control (following the experience with Gantry 1, where the patient table is used as a scanning device). By virtue of the parallelism of the swept beam, a shift of the patient table is equivalent in terms of dose delivery to offsetting the origin of the magnetic scanning (this is not true and not as easy to handle with a divergent beam).

\section{The last $90^{\circ}$ bending magnet}

The last $90^{\circ}$ bending magnet, which directs the beam towards the patient, has been the most challenging element to realize.

The gap of the $90^{\circ}$ bending magnet was chosen at $15 \mathrm{~cm}$ to accommodate for the lateral scanning within the magnet in the $\mathrm{T}$ direction.

In order to fully use the range of $\mathrm{T}$ scanning within the pole gap of this magnet, the vacuum chamber of the $90^{\circ}$ bending magnet has been realized as an integrated vacuum chamber made with non metallic walls, enclosing but not covering the poles of the magnet. The magnet poles thus contribute to the confinement of the vacuum.

Due to the larger gap the last magnet requires also much wider poles as the magnet of Gantry 1. The $90^{\circ}$ magnet of Gantry 2 is therefore heavier and weights 34 tons (much more than the last bend of Gantry 1 which is only 21 tons).

Special attention was paid to the lamination of the $90^{\circ}$ bending magnet, which is machined with separate lamination blocks and with special grooves to limit the effects of eddy currents when working with fast energy changes of the beam.

Due to the stringent requirements to accommodate parallel scanning and to provide fast energy changes, the design of the last bending magnet is the most complex component of the gantry beam line. For further details we refer to [12].

Another peculiar feature of the last bending magnet is the hole in the return yoke, which permits to shine X-ray along the beam axis at the exit of the nozzle (the BEV apparatus described above).

\section{Beam optics properties of the rotating beam line}

In this section we summarize the beam optics properties of the rotating gantry.

The main idea is to inject the beam into the gantry with a rotational symmetric achromatic phase space. The reason of the symmetry is to achieve an invariant beam transport through the gantry independently of the gantry rotation.

\subsection{TRANSPORT calculations}

The beam optics properties of the static beam have been studied by using the program TRANSPORT [13].

Nominal phase space

The nominal values of the injected beam are $\sigma_{x}=\sigma_{y}= \pm 3 \mathrm{~mm}, \theta=\Phi= \pm 9 \mathrm{mrad}, \delta p / p= \pm 0.6 \%$. These values correspond to the beam size used on Gantry 1 [14].

A large transmitted phase space is needed, in view of the use of a cyclotron with a degrader in the beam line ahead of the gantry (see sect. 8.2) in order to keep the beam intensity lost in the degrader as low as possible.

First-order beam optics - beam image

The gantry beam line has been designed as a point-to-point image from the gantry coupling point to the iso-center. 


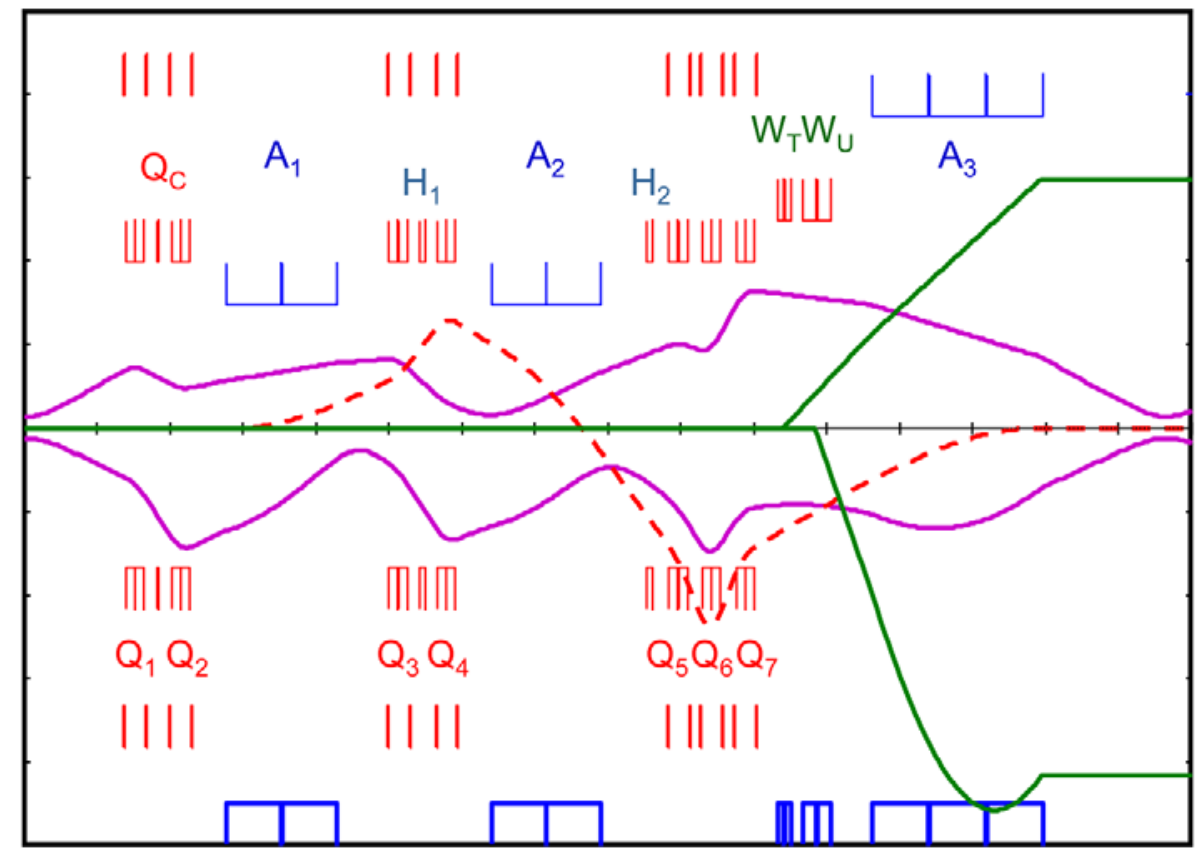

Fig. 7. TRANSPORT beam envelopes trough Gantry 2 (purple). The rays in green represent the action of the sweepers (TRANSPORT code 7). The dotted red line is the dispersion trajectory for a $1 \%$ momentum band. (calculated using the PSI's graphic TRANSPORT framework of ref. [15]).

Table 1. First-order TRANSPORT R matrix from coupling point of the gantry to iso-center.

\begin{tabular}{|c|c|c|c|c|c|c|}
\hline & $1(x)$ & $2(\theta)$ & $3(y)$ & $4(\phi)$ & 5 & $6(\delta p / p)$ \\
\hline 1 & -0.96283 & 0.07927 & 0 & 0 & - & -0.00149 \\
\hline 2 & -0.88350 & -0.96587 & 0 & 0 & - & 0.00062 \\
\hline 3 & 0 & 0 & 0.99881 & 0.00360 & - & 0 \\
\hline 4 & 0 & 0 & -0.04109 & 1.00104 & - & 0 \\
\hline
\end{tabular}

We aim having a focus-to-focus transport system (in terms of the TRANSPORT code, a matrix with R12 = 0 and $\mathrm{R} 34=0)$.

We opted also for a solution with an almost 1:1 imaged size in both transverse directions (a solution close to the conditions $\mathrm{R} 11 \sim \mathrm{R} 22 \sim \mathrm{R} 33 \sim \mathrm{R} 44 \sim 1$ ).

In addition we ask for full achromaticity of the transport system $(\mathrm{R} 16=\mathrm{R} 26=0)$.

These conditions are all nearly satisfied.

In fig. 7 we show the beam envelopes of the original design of the TRANSPORT calculations. In table 1 we give the calculated first-order R1 matrix at iso-center.

The conditions were achieved by fitting using as variables the field strengths in the seven quadrupoles and the entrance and exit tilt angle $\left(10.78^{\circ}\right)$ of the field boundaries of the $58^{\circ}$ bending magnets.

\section{Parallelism of scanning}

Another important requirement is the double parallelism of the scanned beam.

To the first order this condition is equivalent to a point-to-parallel beam transport from the center of each sweeper magnet to the gantry iso-center.

We could achieve this condition by a proper selection of the exit and entrance angles of the $90^{\circ}$ magnet and with a proper choice of the exact location of both sweeper magnets along the beam axis of the gantry. The design values of the entrance and exit angles in the last dipole are $12.2^{\circ}$ and $24.4^{\circ}$.

In the following we discuss the motivations for the chosen specifications.

Expected advantages of using a double small waist at gantry entrance

We have chosen a double focus at the connection point to the gantry for several reasons.

By having a small focus at the coupling point we can afford to have a separation of the vacuum of the gantry (the rotating part) from the vacuum of the rest of the facility (the fixed part). 

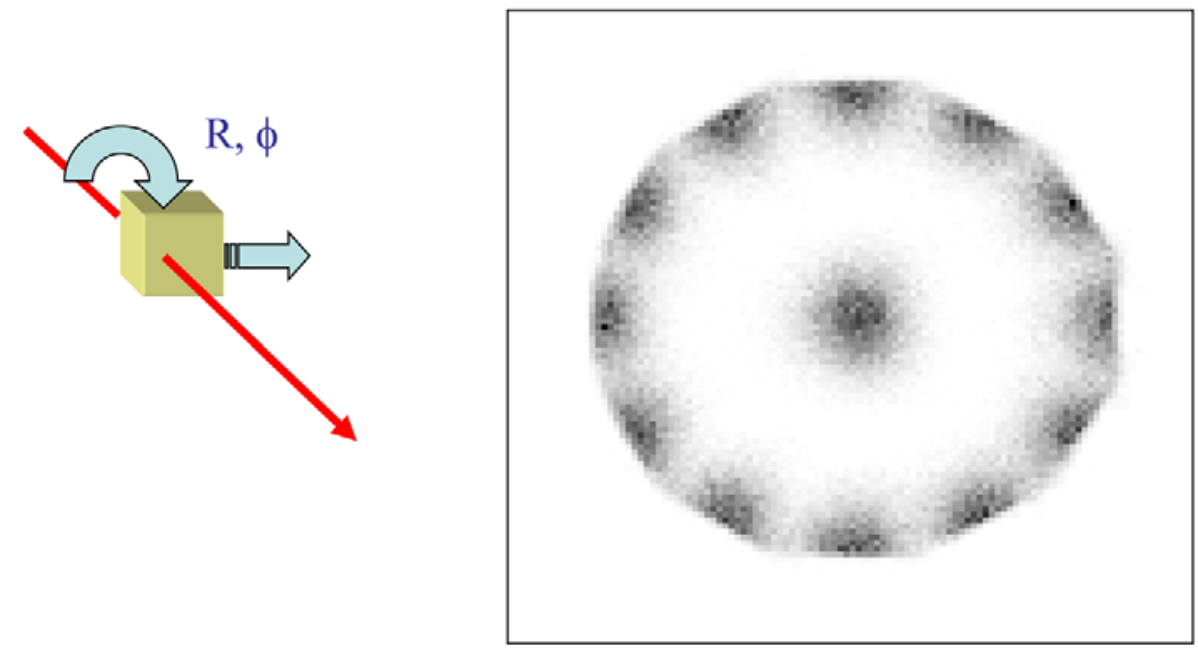

Fig. 8. RAYTRACE calculation (gray shaded image on the right). Randomly chosen rays describing a Gaussian beam are traced from the coupling point to the Gantry 2 iso-center. The large spot in the middle is undisturbed at the center of the scan. The other spots are imaged half Gaussians scanned along a circle. This calculation shows the principle feasibility of an "imaged collimation" of the scanned beam at the coupling point. In practice one could use a rotating and radially moving collimator (left sketch). to create a "virtual collimation" effect on the scanned beam at the border of the target.

The air space region between the two systems is very useful for controlling on-line the quality of the scanned beam. A small amount of material if it is placed at a sharp double focus has very little effect on the final focused image of the scanned beam. By having a region opened to air we can more easily modify the equipment in the beam line at this point. The coupling point is thus an ideal location, where the shape of the scanned beam can be checked ahead of the sweeper magnets. We are presently using at the coupling to the gantry a round collimator of $8 \mathrm{~mm}$ which forces the injected beam to be on the gantry beam axis. We have also an ionization chamber permanently in the beam to measure on-line the beam transmission through the gantry: This detector is also used as a third dose monitor system for safety checks. A fast mechanical beam blocker can shut the beam off in about $60 \mathrm{~ms}$ and is used as part of the safety system. The entrance point to the gantry is therefore a busy location, where we supervise the beam quality before scanning the beam in the gantry.

\section{Expected advantages of using a 1:1 imaged optics}

We expect that such a system is easier to understand and to tune in practice to obtain a small round spot.

By having a 1:1 imaging of the beam optics we can manipulate the size and shape of the beam impinging on the gantry independently of the gantry rotation and of the gantry beam optics. In theory we could apply changes to the beam size at the coupling point ahead of the gantry and expect that these changes are applied directly onto the scanned beam without any need to retune the gantry system. The manipulation of the beam size at the coupling point could be done using the last quadrupole triplet ahead of the gantry ("magnetic change" of the beam spot size ahead of the gantry). To the same purpose we could also use passive devices like scatterers, to create a large Gaussian beam shape of the beam at the coupling point.

Another idea could be to use a dynamic "imaged collimation" applied on such a large scanned beam. A dynamic small collimator moving radially and azimuthally against the beam axis at the coupling point (controlled in function of the scanned beam position at the iso-center), could be used to produce dose distributions with sharp edges at the iso-center using a large beam without the need to mount specific collimators on the nozzle. Figure 8 depicts the principle of this idea. The use of modern computer technology allows performing intensive ray tracing calculations in a short time. The figure shows a scatter plot of randomly chosen particles traced through the Gantry with RAYTRACE (see also sect. 5.2 below). For exploring these possibilities, we would however need to modify the equipment in the air region at the gantry coupling point (i.e. replace the fixed collimator at the coupling point with a more sophisticated solution like for example a moving support holding different collimators with variable apertures). At the moment we decided to postpone these ideas to a later time.

\section{Advantages of using a parallel scanning for therapy}

The advantages of using a parallel scanned beam are manifold. An ortho-normal beam scanning simplifies treatment planning and dosimetry (no source to target distance inverse square law effects affecting the dose distribution). The dose distributions delivered in the patient and in the dosimetric systems are very similar independently of the longitudinal position of the equipment along the beam line. 


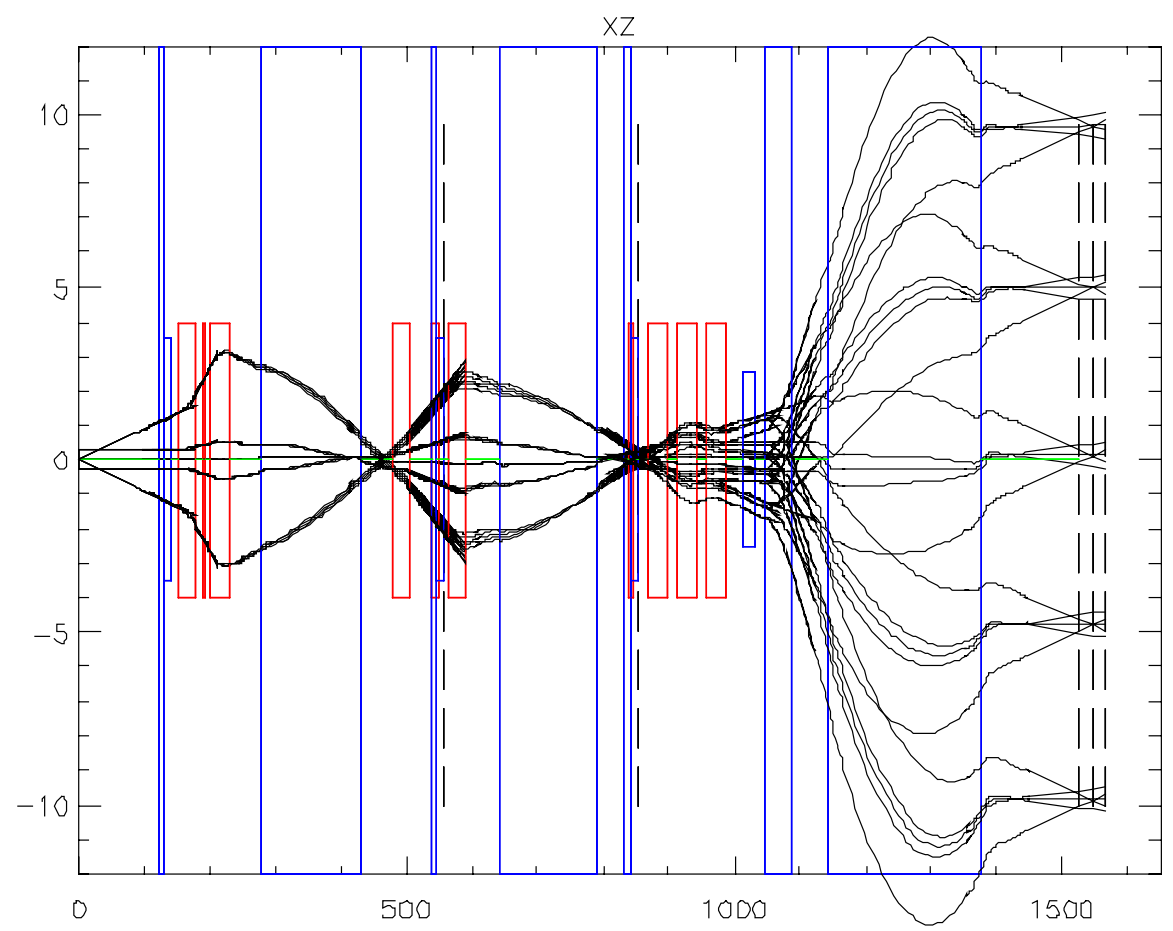

Fig. 9. RAYTRACE calculation. Cosine- and sine-like trajectories in the dispersive plane; for rays of $\pm 3 \mathrm{~mm}$ and $\pm 9 \mathrm{mrad}$. The beam is scanned in the dispersive direction over $\pm 10 \mathrm{~cm}$.

If patching techniques need to be applied by expanding the field size using the motion of the patient table, they are with a parallel beam rather trivial to apply (i.e. without complex optimization calculations within treatment planning as this is needed with a divergent beam). With a parallel beam we have less skin dose, we avoid dose homogeneity errors due the longitudinal dose distortion effects in compensators, collimators can be cut with straight edges etc.

\section{Higher-order beam optics corrections}

The system has been optimized to second order to reduce the optics aberrations to less than $0.5 \mathrm{~mm}$. Without sextupoles the major aberrations are the chromatic ones, T126 $=2.0 \mathrm{~mm}$ and T346 $=1.5 \mathrm{~mm}$. It is not clear if these aberrations need to be corrected in practice. We could not yet observe with the beam a clear visible effect on the beam shape using the sextupoles. The sextupoles are therefore at the moment not used in practice (zeroed).

\subsection{Beam optics calculation with RAYTRACE}

The beam optics calculations for studying in more details the properties of the scanned beam were done with the program RAYTRACE [16], because TRANSPORT being based on Taylor expansions does not support beam calculations far away from the nominal trajectory, which is the case with a scanned beam. We decided to use the same code which was successfully used for Gantry 1 in 1993 [17]. With this approach single particle trajectories are calculated through (idealized) three-dimensional analytical magnetic field maps representing the various beam line elements.

Figure 9 shows selected rays along the beam line as a plot of the distance of the rays from the central trajectory (images obtained by interfacing the output files of RAYTRACE to an own developed program with graphical interface for plotting the results). We show the cosine- and sine-like trajectories in the $x-\theta$ plane projected on the dispersive plane (rays with $\pm 3 \mathrm{~mm}$ and $\pm 9 \mathrm{mrad}$ ).

Figure 10 shows the cosine- and sine-like trajectories for the transverse plane $y$ - $\varphi$. The beam is scanned transversely within the gap of the $90^{\circ}$ bending magnet over $12 \mathrm{~cm}$.

\section{Orthogonal focal planes of the scanned beam}

A major goal of the beam optics design was to obtain a small beam size of the scanned beam, which is well focused and does not vary much in depth depending on its position along the $\mathrm{T}$ and $\mathrm{U}$ plane of scanning. To obtain this we must keep the beam well focused independently of scanning, despite the fact that the beam goes through quite different regions of the $90^{\circ}$ bending magnet and has different path lengths. We aim at having a sharp $x$ - and $y$-focus in the whole $\mathrm{T}$ and $\mathrm{U}$ scan region at the iso-center. In the language of spectrometers we look for $x$ - and $y$ - orthogonal focal planes. 


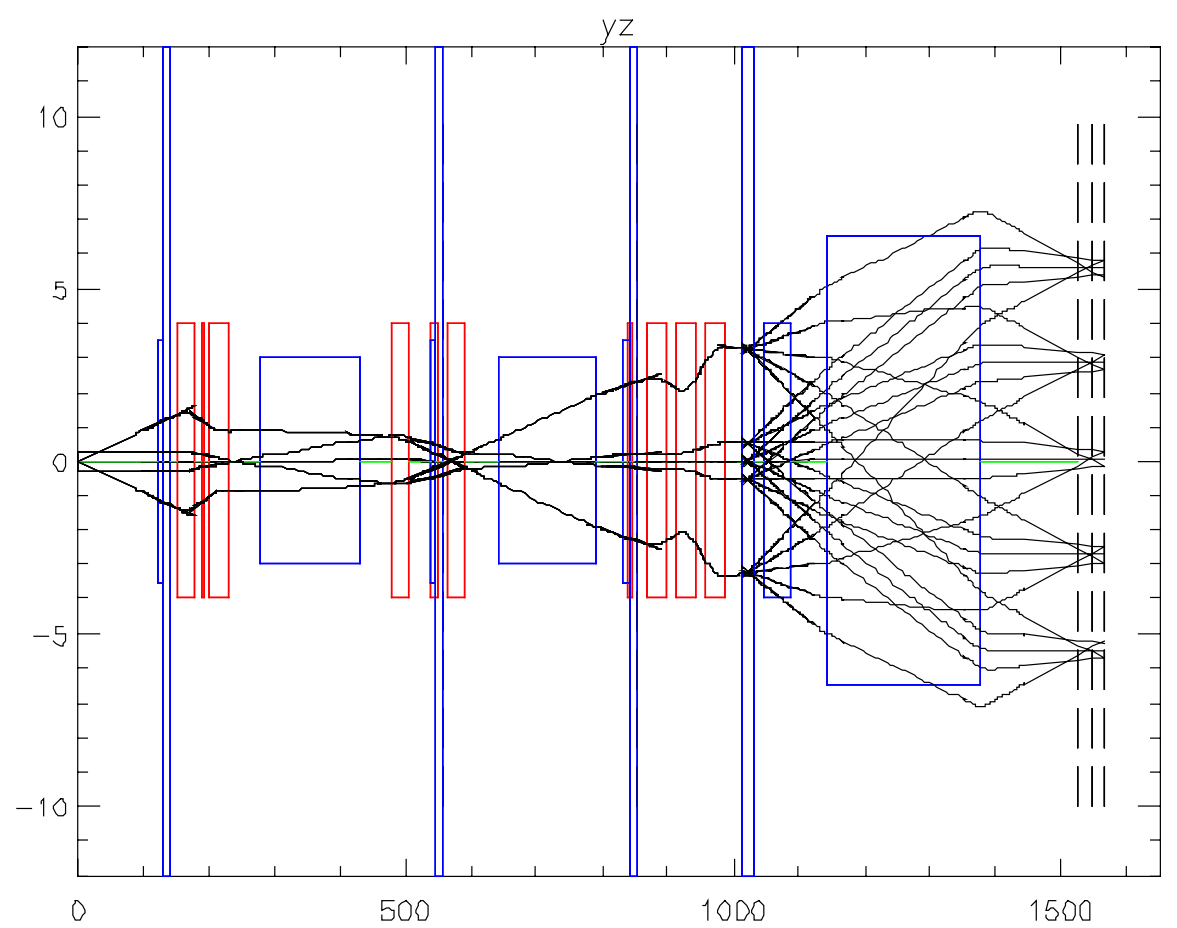

Fig. 10. RAYTRACE calculation. Cosine- and sine-like trajectories in the transverse plane; for rays of $\pm 3 \mathrm{~mm}$ and $\pm 9 \mathrm{mrad}$. The beam will be scanned transversely within the gap of the $90^{\circ}$ bending magnet over $\pm 6 \mathrm{~cm}$.

With Gantry 1 [17] it has been possible to have a scanning-invariant focus by using only passive corrections $(i . e$. corrections embedded in the design of the iron of the magnets), but the task was much easier at that time since the scanning of Gantry 1 is applied only in one dimension, the dispersive U direction. With Gantry 2 we have added another scanning dimension $(\mathrm{T})$ to the problem. The number of degrees of freedom available on the very compact beam line of Gantry 2 is not sufficiently large for solving the problem using only passive correction elements.

A tilt of the $x$-focal plane as a function of the U-sweeper was corrected by applying a tapering to the poles of the U-sweeper magnet (the same kind of correction, which was used for Gantry 1). Within the U-sweeper gap the field decreases by $7 \%$ over $10 \mathrm{~cm}$ in the direction of the larger gantry radius. This feature corrects for a longitudinal displacement of the $x$-focus ranging over about $\pm 20 \mathrm{~cm}$ when the beam is scanned laterally over $\pm 10 \mathrm{~cm}$ in the $\mathrm{U}$ direction (suppression of the tilt angle of the $x$-U-focal plane). For the $y$-U focal plane we observed an effect of similar magnitude in the RAYTRACE calculations. We could not find a passive correction for this effect. We therefore decided to insert a thin quadrupole $\mathrm{Q}_{\mathrm{C}}$ placed in the middle of the first quadrupole doublet of the gantry. We have realized in the end in practice this correction by connecting the cables of the quadrupole corrector $\mathrm{Q}_{\mathrm{C}}$ in series with the U-sweeper. As discussed below, this correction appears to be quite important in practice.

\section{Nozzle design for achieving a small beam size}

Figure 11 shows the layout of the nozzle.

The practical construction of the nozzle is shown in fig. 3 .

\section{Vacuum extension}

The vacuum chamber of the last bending magnet extends into the nozzle in order to minimize the space where the beam goes through air and to reduce the distance of the vacuum window to the iso-center, which is in this case about $90 \mathrm{~cm}$. The main goal of the design is to minimize any beam broadening of the pencil beam due to scattering in the material and air in the nozzle.

\section{Beam monitors}

Within the nozzle we mounted two transmission monitors M1 and M2. These plane parallel ionization chambers measure the instantaneous intensity of the pencil beam and are calibrated as number of delivered protons. They are used to control on-line the amount of delivered dose.

A strip monitor (ionization chamber with $2 \mathrm{~mm}$ wide strips in the $\mathrm{T}$ and $\mathrm{U}$ directions) is mounted in the same box (the chamber has been fabricated and delivered to PSI by the University of Torino in the framework of a collaboration 


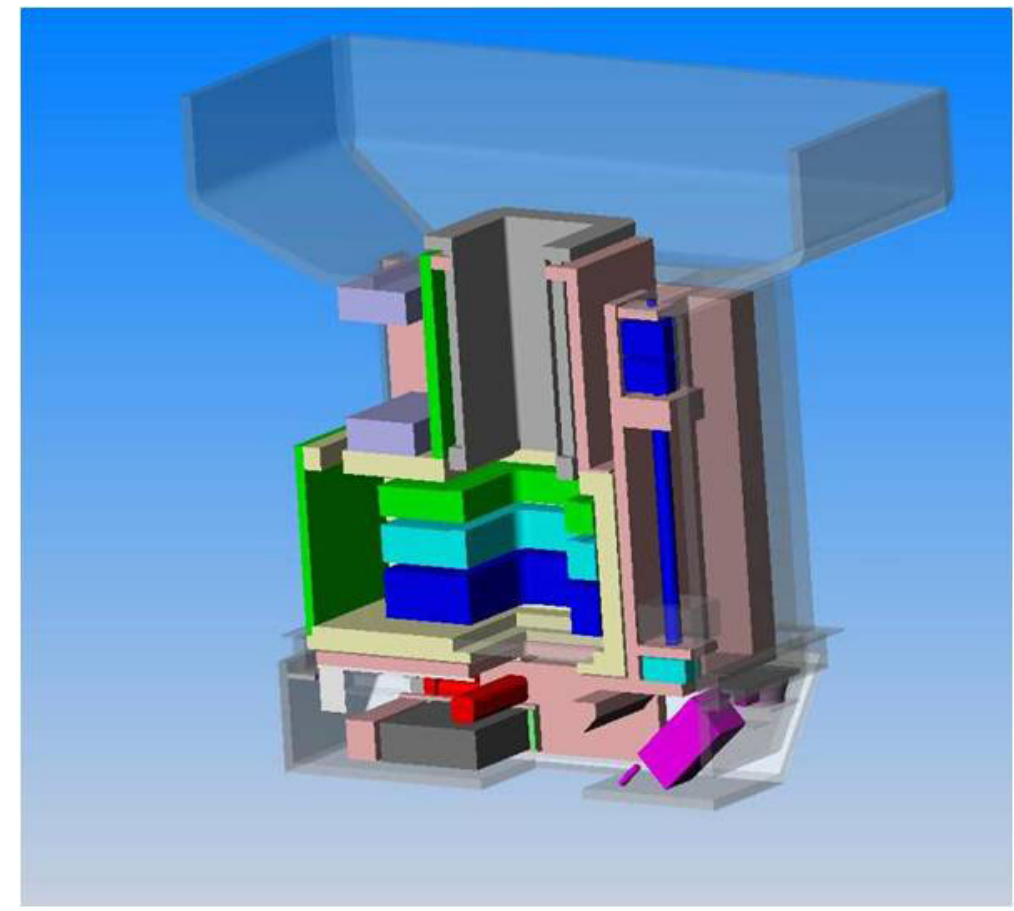

Fig. 11. Components of the nozzle of Gantry 2. From top to bottom we see the extension of the vacuum into the nozzle (upper gray box). Underneath of this we find the two transmission ionization chambers (green and pale blue), the strip monitor (dark blue). Below the box containing the monitors we have a preabsorber block, which can be moved under remote control into the beam. In this region we have also lasers and TV cameras. The whole nozzle can be moved longitudinally and is enclosed by collision-detecting panels.

with the TERA group in Italy). This monitor is used for measuring the position and shape of the pencil beam in front of the patient during beam delivery. These monitor elements are contained in an insulated box, attempting to provide an optimally shielded environment against electrical noise, sound and mechanical vibrations.

\section{Removable pre-absorber block}

Underneath the detector box we have a support, which allows moving under remote control a block of graphite (with thickness corresponding to about $8.4 \mathrm{~cm}$ water equivalent range) in and out of the beam. This block will be used for delivering in the patient the proton ranges, which are not available in the beam tune range (i.e. energies below $100 \mathrm{MeV}$ when scanning with continuous lines painting with dynamic modulation of the beam intensity and energies below $70 \mathrm{MeV}$ when using discrete spot scanning, see sect. 8.2).

\section{Laser and TV camera}

At the same height level of the pre-absorber block, we have a TV camera and a set of lasers mounted laterally outside of the scan area, which will be used for viewing the alignment of the patient on the beam axis from within the nozzle. These elements gaze, respectively shine, through a transparent foil, which encloses the exit part of the nozzle. The nozzle is completely enclosed with collision detection plates to guarantee patient safety against mechanical injury.

\section{Longitudinal nozzle motion}

The whole nozzle, including the monitors and the elements below them, can be moved along the beam direction under remote control over a distance of $27 \mathrm{~cm}$. We plan to use this motion to reduce the air gap between patient and nozzle, while keeping the tumor and the patient at the iso-center, since this position is the place where the beam is best focused.

\section{Collision detection}

The whole gantry system is protected against mechanical collisions by a comprehensive collision detection system. In this way we will be able to move the gantry and the patient table and the elements in the nozzle under remote control. It will be thus possible to deliver several fields in sequence without personnel entering in the room, following the established experience of Gantry 1. 


\section{Option to add collimators and compensators}

Underneath the pre-absorber block we have a support, on which we can mount compensators and collimators. On Gantry 2 it will be possible to investigate the possibility to add collimation on top of scanning, which could be beneficial for improving the lateral dose fall-off at very low energies. In addition we plan to show that a gantry system can rather effectively simulate the passive scattering technique, without the need of fabricating scattering foils and range shifting propellers.

\section{Nozzle optimization for a small beam spot}

The nozzle has been designed as compact as possible longitudinally in order to be able to easily position the patient very close to the nozzle with a minimal air gap. The nozzle compactness in the transverse directions is on the other side also important for minimizing the air gap by avoiding collisions of the nozzle for example with the shoulders of the patient.

\section{Main features of the beam line system ahead of Gantry 2}

The layout of the beam line to the Gantry 2 within the PROSCAN facility is shown in fig. 2 . The design of the beam line before the gantry is of equal importance for the performance of the whole system as the gantry itself.

In this section we describe the main components, which play an important role for using the Gantry 2 system. The specifications of the new expanded facility PROSCAN were defined in view of the new development goals of Gantry 2.

\subsection{The $250 \mathrm{MeV}$ superconducting cyclotron}

At the origin of the beam line we have a $250 \mathrm{MeV}$ superconducting cyclotron.

The intensity of the beam can be chosen (as a slow change) at the ion sources in a range from a few nA up to $850 \mathrm{nA}$ proton current. For Gantry 2 the average used proton current is around 200-400 nA.

Main attention was given to the stability of the beam. The cyclotron delivers a stable DC beam best suited for pencil beam scanning. The original specifications aim at a stability of the beam intensity of a few $\%$ standard deviation at the time scale of $100-200 \mu$ s (and slower)

The beam stability of the source is of importance for painting the dose with dynamic modulation of the intensity of the beam (see sect. 8.7).

\subsection{Vertical deflector plate}

In the central region of the cyclotron in the first turns of the beam after the ion source we have a vertical deflector plate. By varying the electric field we can deflect the beam such as to reduce the beam transmission in a set of collimators along the first orbits of acceleration. In this way we can vary the intensity of the extracted beam (as a continuous variable) extremely quickly (from 0 to $100 \%$ in about $0.1 \mathrm{~ms}$ ). The dynamic modulation of the beam intensity will be used for giving shape to the dose distribution when moving the beam with maximum velocity along lines, planes and contours.

\subsection{Kicker magnet}

Immediately after the cyclotron we have a quadrupole triplet which focuses the beam on a small collimator hole located at the beam entry point to the next important section of the beam line, which is the degrader system. Following the quadrupole triplet we have a kicker magnet, which can displace the focused beam in and out of this collimator hole.

The kicker is used to switch the $250 \mathrm{MeV}$ beam on and off as desired. The kicker acts as a fast switch with a $50 \mu \mathrm{s}$ beam off reaction time and up to about $1 \mathrm{kHz}$ repetition rate capability and is used by tradition at PSI for the delivery of the discrete spot scanning method developed on the old Gantry 1 (see also sect. 8.7 below).

For compatibility reasons the kicker will be used in the same way also with Gantry 2 but the deflector plate could be used as well for switching the beam on and off on a spot by spot basis as a faster alternative and/or as a redundant switching system.

The kicker system permits in addition to control on-line the quality of the beam extracted from the cyclotron, before the kicker is opened and the beam is sent into the beam line to the gantry for treatment delivery. 


\subsection{Degrader}

The degrader consists of two opposed sets of carbon wedges, which are moved mechanically very quickly in and out of the beam. The energy can be changed in the range between $70 \mathrm{MeV}$ and $230 \mathrm{MeV}$ (a fixed value of $250 \mathrm{MeV}$ is also possible but was not yet tried). The time to change the position of the wedges for a small energy change corresponding to about $5 \mathrm{~mm}$ proton range in water is within $50 \mathrm{~ms}$.

The degrader system is contained in a vacuum box as a part of the vacuum system of the beam line.

After the carbon wedges we have two sets of round collimators (selectable from a series of collimators mounted on a moving tower) which define the size (the first collimator) and the angular acceptance (second collimator) of the beam emerging from the degrader. The second collimator is mounted just before the next quadrupole doublet. For Gantry 2 we have chosen among the available collimators those which gave the closest agreement to the accepted phase space at the coupling point of the Gantry 2 .

\subsection{Achromatic beam line section with momentum analysis}

The beam line following the degrader consists of two sections.

The first section is a double bend symmetric achromat, with a horizontal focus at the symmetry point. A slit system at this location analyzes the energy content of the beam. By setting the slit jaws position (in vacuum) we can select the amount of momentum band, which is further transported in the beam line. It is important to note that, due to multiple Coulomb scattering (MCS) and energy straggling in the degrader, a lot of beam is lost in the collimators in the degrader region of the beam line. The amount of these losses depends strongly on the amount of energy degradation applied. As an example the beam transmission changes between $230 \mathrm{MeV}$ at $70 \mathrm{MeV}$ by about two orders of magnitude.

\subsection{Beam line connection to the Gantry 2 with several intermediate collimators}

Following the beam analysis section, we have another section, which is used for diverting the beam into three different areas (see the layout of fig. 2). In the beam line in direction to Gantry 2 we focus the beam at another intermediate double focus. At this location we decided to interrupt and separate the vacuum in the same way as this is done at the coupling point to the Gantry 2, which is further downstream. This intermediate focus is located before the shielding wall, which separate the beam switchyard from the gantry area. At this first double focus point we have a round collimator with an opening of $8 \mathrm{~mm}$ diameter. This collimator in air provides the primary compensation of the intensity losses described below in sect. 8.2.

Before the separation wall we have mechanical beam blocking devices. The beam traverses the separation wall to the Gantry 2 and is focused with a triplet at the coupling point to the gantry to obtain a rotational symmetric double focus with phase space values, which match the nominal values of the gantry.

\section{Preliminary commissioning results}

In this section we report on our first commissioning experience.

\subsection{Dealing with the impregnation problems of the $90^{\circ}$ bending magnet}

Figure 12 shows the $90^{\circ}$ bending magnet being assembled with integrated vacuum chamber.

Since the poles of the $90^{\circ}$ bending magnet are used for the vacuum confinement, the pole surface of the magnet were vacuum-impregnated with epoxy resins in order to be vacuum tight. Due to initial fabrication difficulties we had at the beginning vacuum leaks in the lamination of this magnet: The pole surfaces were re-machined, polished and retreated with glue at PSI by the vacuum and the magnet group of PSI. This problem was an unexpected difficulty encountered in the realization of a first prototype by attempting to use a new technology. The problem produced a significant delay in the project time table. Due to the engagement of the PSI experts, very good vacuum conditions were nevertheless achieved in the end and the $90^{\circ}$ bending magnet was successfully installed on the gantry. Since then (2008) the system behaves perfectly, with vacuum values down to $10^{-6}$ mbar (much better than required). 


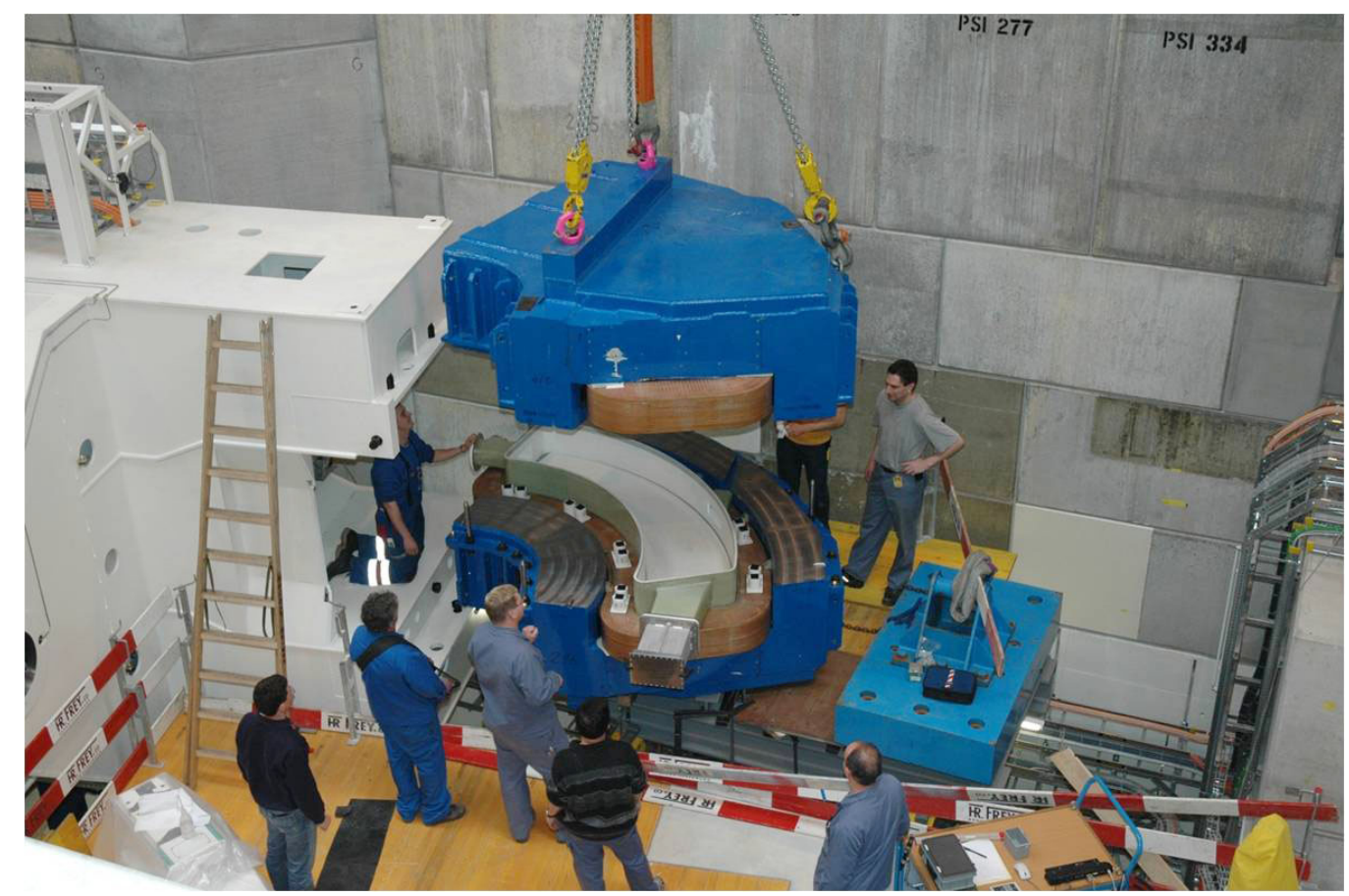

Fig. 12. Photograph of the $90^{\circ}$ bending magnet being mounted on the Gantry 2. A very interesting detail is the integrated vacuum chamber embracing the poles of the magnet.

\subsection{Initial tuning of the whole beam line from the accelerator to the gantry}

We report in this section on how we provided a first solution for tuning the Gantry 2 with variable beam energy.

To this goal we developed own software tools, which allow tuning the beam line interactively on-line, while sending the results of instant TRANSPORT and TURTLE calculations (using the TRANSPORT code version of Fermilab [18]) to the control system of the beam line, to investigate the actual behavior of the beam. Beam optics input files for the whole beam line were generated as a function of the beam energy on-the-fly and sent to TRANSPORT and TURTLE using an own developed $\mathrm{C}^{++}$program with a graphical interface. The beam optics calculation results were displayed as plots (fig. 13) and the corresponding current values for the magnets were sent within a single command to the machine control system. The code was further expanded with embedded polynomial expressions which parameterize step by step the intermediate results of the tuning process. In the end an optimized piece of code describing the data for the whole beam line system for all beam energies was produced. This part of the code containing all fit results was finally integrated in the treatment delivery software (as a part of the so-called beam tunes generator).

Where applicable the default approach in the tuning process has been to apply simple momentum scaling for the energy dependence (this corresponds to the same beam optics solution for different energies). This approach is however not optimal in terms of transmitted beam intensity. If we apply simple scaling, we have big variations in the beam intensity due to the varying losses in the degrader as a function of the beam energy (with changes of up to two orders of magnitude). TRANSPORT itself does not support the handling of beam losses in the degrader and in collimators as a function of the energy. This is why we had to use TURTLE as well.

We divided in the code the beam line into four sections: section 1 before the degrader, section 2 from the degrader to the (second) "beam-suppression collimator", section 3 from this intermediate collimator to the gantry coupling point and as section 4 the gantry beam line itself.

As the first step, we assessed the intensity losses and the size of the phase space of the beam emerging from the degrader for different degraded energies. This was done by fitting Monte Carlo results describing the phase space of the beam at the exit of the degrader obtained with the code SRIM [19]. With this parameterization in place we could run TURTLE through the whole beam line of section 2 including the action of the collimators in this part of the beam line. 


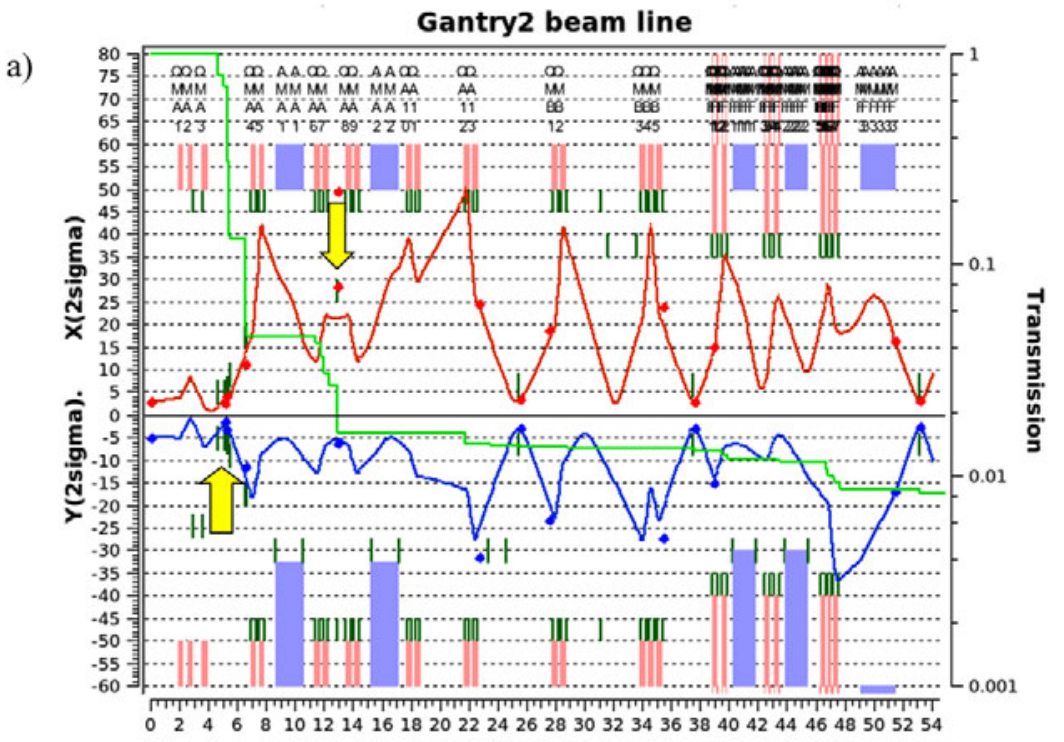

Gantry2 beam line

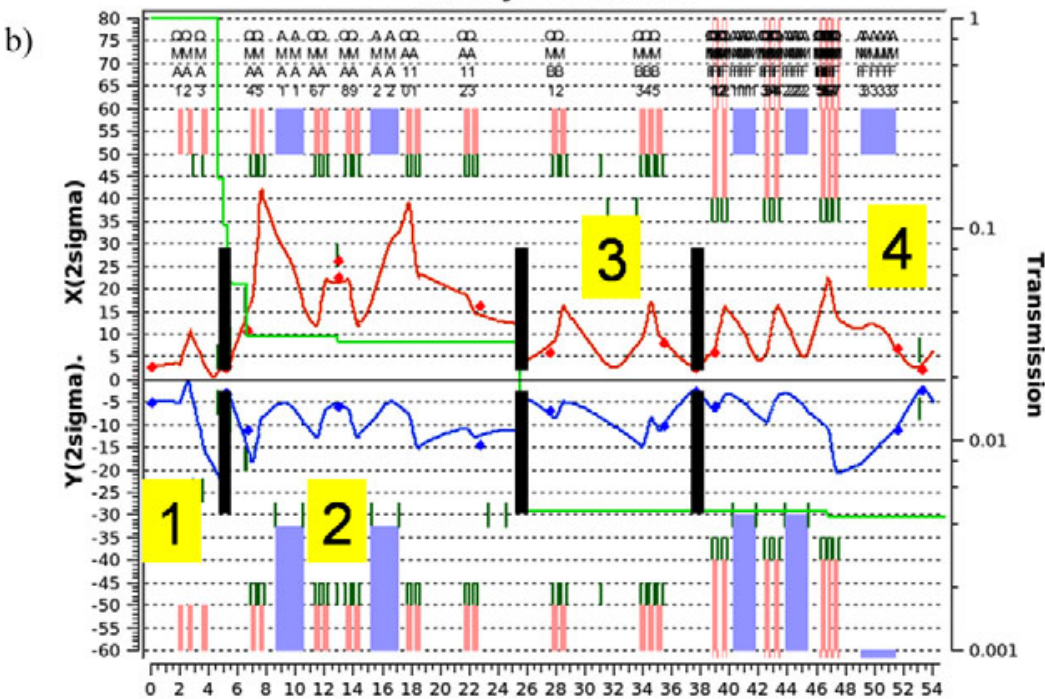

Fig. 13. a) Beam envelopes at $100 \mathrm{MeV}$. Horizontal (red) and vertical (blue) envelopes (left scale). The green line is the transmitted beam intensity and the dots are beam sizes calculated with TURTLE. Most of the beam is lost at this energy in the degrader (the first arrow) and in the momentum analysis slit (the second arrow). b) Beam envelopes at $200 \mathrm{MeV}$. In yellow we delineate the four beam line sections described in the text. The beam is defocused at collimators (black bars) The beam major suppression is at the degrader entrance and at the second collimator. In this way we obtain the same intensity losses as with the $100 \mathrm{MeV}$ solution of panel a.

\subsubsection{Tuning with compensated intensity losses in the range from 100 to $200 \mathrm{MeV}$}

At this point we decided to optimize the whole beam line including the sections 3 and 4 for maximum transmission at a fixed reference energy of $100 \mathrm{MeV}$. At this energy the beam is well focused on the 8 mm collimators at the end of the sections 2 and 3 and is optimized for maximum transmission through the whole beam line system.

We then did run TURTLE through the whole system with different energies (by using momentum scaling). In the range between 100 and $200 \mathrm{MeV}$ we then provided an equalization of the intensity losses by intentionally defocusing the beam at the intermediate collimator at the end of section 2 . We intentionally stop part of the beam in the collimator by detuning the last quadrupole doublet in front of the collimator as a function of the beam energy. The achieved beam suppression effect was however not sufficient to provide a complete equalization of the beam intensity. A restriction was due to the radiation protection limits, which apply in this region of the beam line, since the shielding was not planned for beam losses at this place. We therefore decided to apply a similar beam defocusing effect also at the first 
focus at the entrance of the degrader. The defocusing is applied there only in one of the transverse directions in order not to affect the functionality of the kicker.

The results of TURTLE calculations were iteratively modeled and the tuning was optimized also in view of the experimental work with the beam. Fits were derived for describing the reduced phase space of the beam emerging from the second collimator at the end of section 2 as a function of the beam energy. The "intensity-equalized beam" was then further transmitted through the rest of the beam line in sections 3 and 4 . In the end a solution with full beam intensity equalization in the range between 100 and $200 \mathrm{MeV}$ could be found.

Figure 13a shows a TRANSPORT calculation through the whole system at $100 \mathrm{MeV}$, optimized for full transmission through the whole beam line. In fig. 13b one can recognize how the beam is defocused at $200 \mathrm{MeV}$ in a controlled way at the entrance of the degrader and at the end of section 2 . The green line shows the beam intensity along the beam line calculated with TURTLE and SRIM.

With this strategy we can now keep constant the beam transmission through the whole beam line from the accelerator to the patient independently of the beam energy.

We can change the beam energy as a continuous variable.

When we change the beam energy we modify only the settings of the power supplies of magnetic devices of the beam line, which are fast. The values of the slow mechanical devices like the slits jaws remain unaffected. The only exception is the degrader, which moves mechanically but moves also very fast. In this way we can provide very fast energy changes, with constant beam intensity at the gantry iso-center, independently of the settings in the cyclotron unit.

This mode of beam tuning is planned to be used for developing dose painting with continuous line scanning with modulation of the beam intensity. The major reason to tune the beam with equalized intensity is to have a solution, where the nominal beam intensity at the patient is kept constant independently of the beam energy. Since we do not use the deflector plate for compensating beam intensity losses in the degrader, we can dedicate its use fully for dose painting. We thus can provide fast beam intensity changes, with a large dynamics in the intensity range, independently of the beam energy.

We could not extend further the intensity compensation above $200 \mathrm{MeV}$ due to insufficient shielding in the region of the second intermediate collimator in air at the end of section 2 . If needed, this could be corrected in the future by adding local shielding at this intermediate collimator.

One could also as an alternative provide intensity compensated tunes at higher or lower energy intervals, for example between 70 and $150 \mathrm{MeV}$ and 130 and $230 \mathrm{MeV}$.

This could be future commissioning work.

For the moment we plan to use a $8.4 \mathrm{~cm}$ water equivalent pre-absorber block (a $\sim 5 \mathrm{~cm}$ thick carbon plate) for delivering beam energies below $100 \mathrm{MeV}$ in the patient. We thus have a first basic solution for exploring the use of new advanced scanning techniques (based on the idea to scan the beam continuously as a function of the real time at high sweeper speed in combination with the use of the dynamic modulation of the beam intensity for shaping the dose distribution) (see sect. 8.7 below).

\subsubsection{Tuning for discrete spot scanning in the range $70 \mathrm{MeV}-230 \mathrm{MeV}$}

The use of the vertical deflector plate is not needed for the delivery of discrete spot scanning (discrete spot scanning can be applied without a precise control of the instantaneous dose rate of the beam). For this beam delivery mode we have extended the available energies — below $100 \mathrm{MeV}$ down to $70 \mathrm{MeV}$, and above $200 \mathrm{MeV}$ up to $230 \mathrm{MeV}$ by applying a simple momentum scaling to the beam optics from the two boundary energies. In the extended beam energy region we further adjusted the intensity of the beam with the deflector plate, which is then set by default as a tune-specific command. We can thus almost equalize the monitor unit rate in the whole range from $70 \mathrm{MeV}$ to $230 \mathrm{MeV}$.

At the lowest energy we can change the extracted intensity up to about $850 \mathrm{nA}$. This should allow keeping the beam delivery time short also for dose fields with very low penetration depths.

\subsection{Mapping of the action of the sweeper magnets on the scanned beam}

To first order one expects a linear relationship between the sweeper magnets current and the scanned beam position with a good preservation of the parallelism of the scanned beam. In practice we found significant non-linearities already within the calculations with the idealized magnetic field maps of RAYTRACE (not containing saturation effects). The effects are however much bigger with the real magnets due to local saturation effects. In practice we found that it is absolutely necessary to map the position of the scanned beam as a function of the current in the sweeper magnets on the basis of experimental results performed with the beam (with an individual map for each energy).

Before mounting them the beam line elements were checked by the magnet group of PSI by providing magnetic field map measurements. The magnetic field maps for the last bending magnet of Gantry 2 showed relevant saturation effects at the corners of the poles. The saturation depends on the applied magnetic field and therefore these effects 

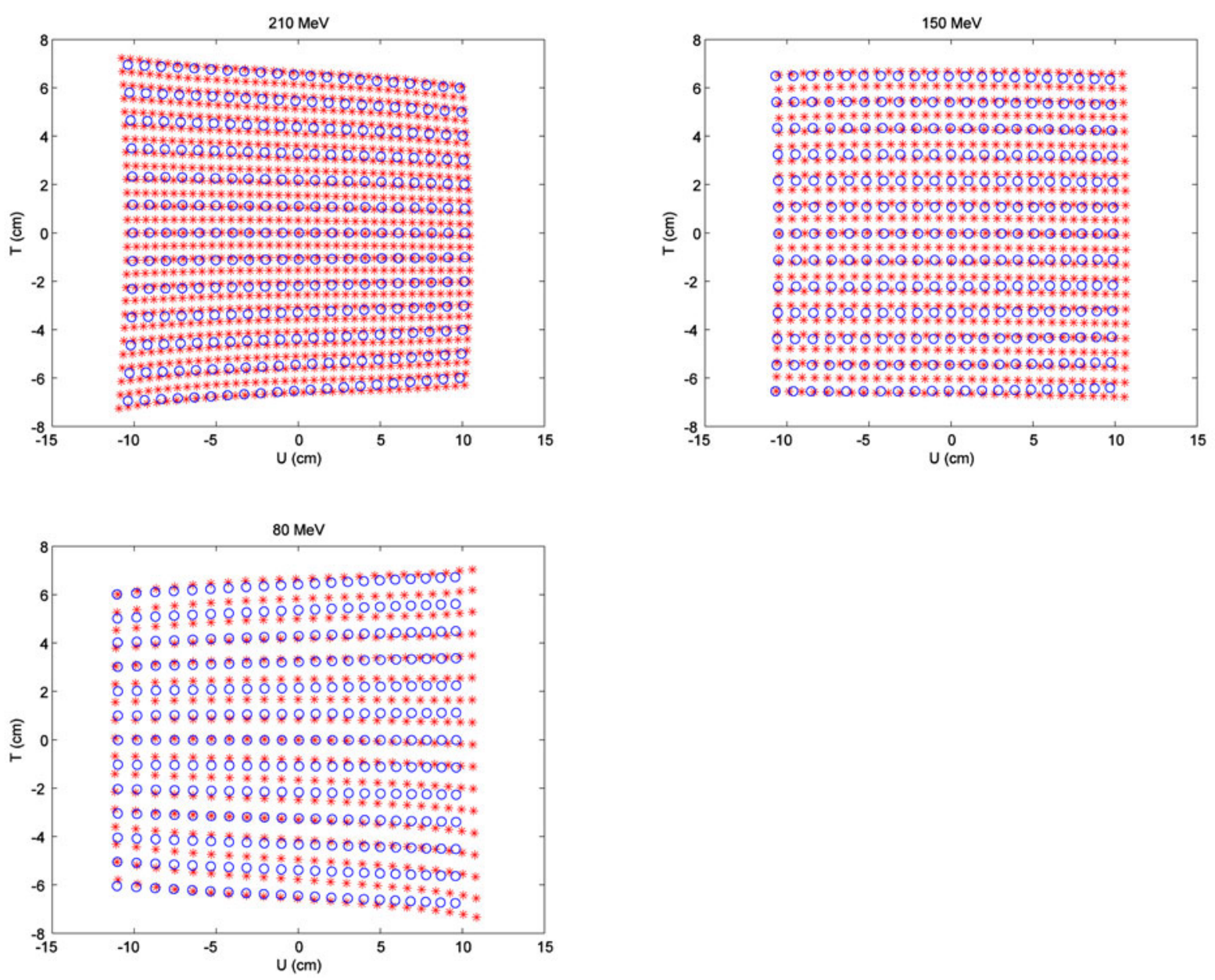

Fig. 14. Measured (red) and calculated (blue) spot maps of the action of the sweeper magnets on the scanned beam position. The qualitative agreement indicates that the major distortions are due to the curvatures of the effective boundary of the magnetic field of the $90^{\circ}$ bending magnet.

change with beam energy. The poles of the last $90^{\circ}$ bending magnet were specified by design with a straight entrance and exit edge. A straight exit edge is desired for having a vertical focus at the iso-center independently of the setting of the U sweeper. The measured effective magnetic field boundaries of the $90^{\circ}$ bending magnet differ significantly from the idealized fields in the RAYTRACE calculations and also from the mechanical shape of the poles. The most prominent effect affecting the scanning maps is due to an effective field edge curvature at the entrance and exit edge of the poles of the last dipole, which instead of being of zero inverse radius as desired by design, changes from $-0.4 \mathrm{~m}^{-1}$ to $+0.4 \mathrm{~m}^{-1}$ in the energy range from $70 \mathrm{MeV}$ to $230 \mathrm{MeV}$. These effects (especially the one at the exit face of the last dipole) produce remarkable distortions in the orthogonality of the scan. We show as an example the effect of the measured exit field curvatures on the scan pattern in fig. 14 for $210 \mathrm{MeV}, 150 \mathrm{MeV}$ and $80 \mathrm{MeV}$, respectively. The spot positions were calculated with RAYTRACE by using effective field edge parameters derived from the measured magnetic field maps. The calculation points were derived by scanning the beam along an equidistant grid of magnetic field values of the sweepers. The beam positions resulting from the RAYTRACE calculations are plotted in the figures as the blue circles. The symbols in red are the measured positions of the beam at the iso-center of the gantry obtained by changing the sweeper's values on a regular grid, in terms of the current of the power supplies. The qualitative agreement between calculation and experiment for different beam energies indicates that the curvature at the exit magnetic field edge of the last bending magnet is mainly responsible for the observed distortions in the beam position maps of the sweepers.

From the calculations we could anticipate that the curvature at the field edge has similar effects also on the parallelism of the field. The errors in the beam direction vary in average around a couple of mrad. The worse effect is at the highest energy, where at the corner of the scan area a distortion up to 5 mrad is expected. With Gantry 2 we plan to treat the patient always with the center of the tumor located at the iso-center. The error due to the "wrong" parallelism of the beam is thus expected to be below $\pm 1 \mathrm{~mm}$ over a maximal longitudinal tumor size of $\pm 20 \mathrm{~cm}$, what should be practically acceptable. 

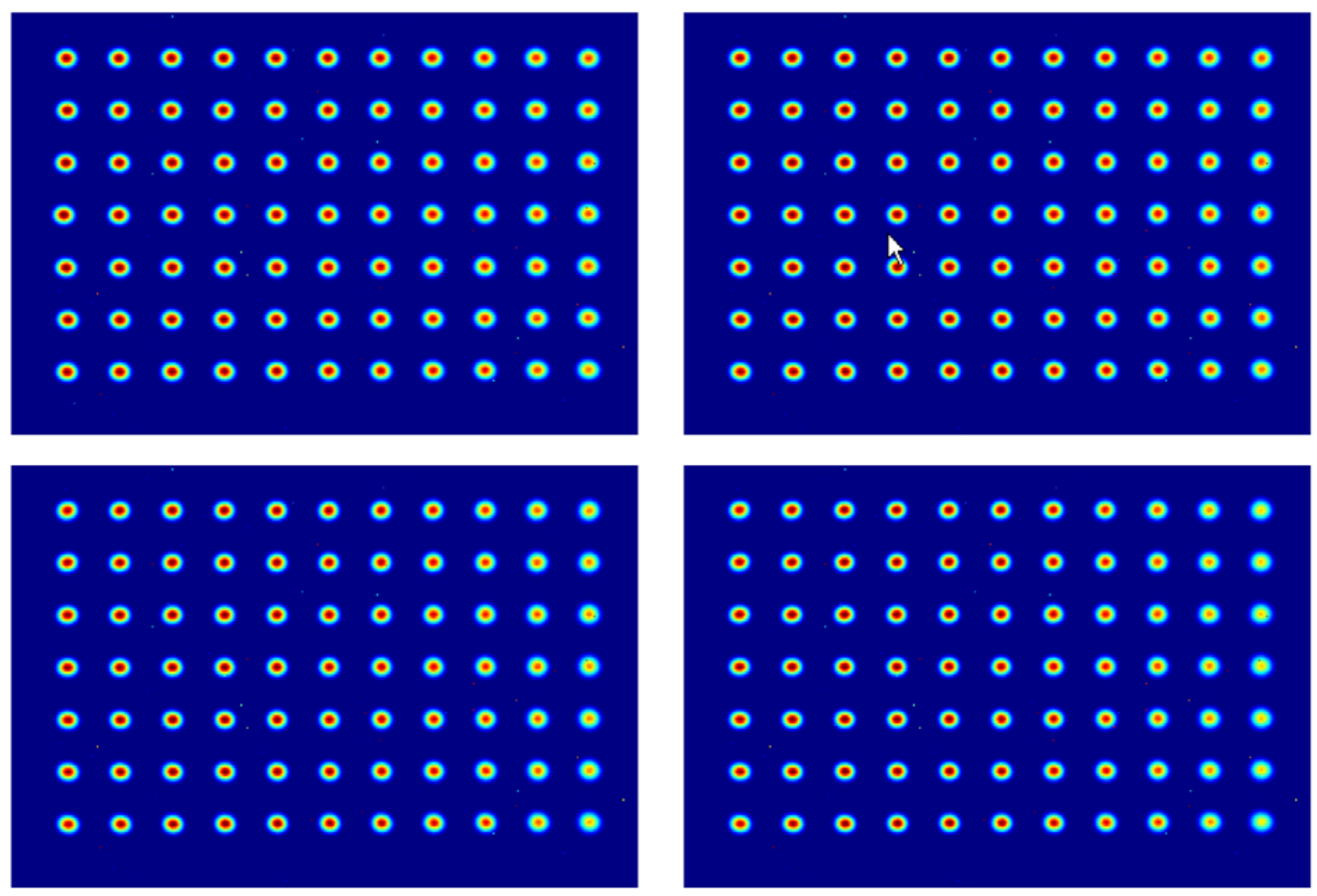

Fig. 15. Image of the beam spots at the isocenter covering the scan region of $12 \mathrm{~cm} \times 20 \mathrm{~cm}$ for $70 \mathrm{MeV}$ (top left) $120 \mathrm{MeV}$ (top right) $170 \mathrm{MeV}$ (bottom left) and $220 \mathrm{MeV}$ (bottom right) after a proper calibration of the sweepers.

We believe that for practical purpose it will be possible to work within treatment planning as if the beam would be perfectly parallel. We will perform more precise measurements on the beam parallelism as soon as we will be able to change the distance of the detector from the nozzle using the remote control of the patient table.

With the realization of a new magnet for a new gantry of the same design, one could presumably reduce the exit field curvature effects of the last bending magnet by adding correction coils onto the poles (as a tunable sextupole component), which could be energized as function of the beam energy. It is not clear to us yet, if the benefit of such a correction element is really worth the effort of being realized, due to the added complexity and costs of the extra equipment in practice. This is a possible point to discuss in the future with magnet technology experts.

The use of an experimental parameterization of the effect of the sweeper magnets on the beam position at the iso-center appeared necessary already with the idealized field maps of RAYTRACE. As a practical approach to this problem we provided in the end experimentally derived sweeper maps of the position of the beam at the iso-center for a full set of beam energies in steps of $10 \mathrm{MeV}$. The single energy maps were fitted with polynomials as to provide the current values of the U- and T-sweeper magnets for any given U, T position of the scanned beam. Intermediate energy maps (within the $10 \mathrm{MeV}$ intervals) are scaled in momentum and interpolated between adjacent energy maps. Figure 15 shows some results of using the final scan maps while scanning the beam in steps of $2 \mathrm{~cm}$ over the nominal scan range of $20 \mathrm{~cm} \times 12 \mathrm{~cm}$. The data are dose images of the beam impinging on a scintillating screen seen by a CCD camera $[20,14]$.

The results look at the end rather satisfactory.

By using a proper (smooth) mapping of the swept positions of the beam (using polynomial expressions), we linearized the action of the sweepers through software. In this way we retrieve not only a good fidelity of painting the border of the dose distribution to the shape of the target but we eliminate as well all disturbing effects which could affect the homogeneity of the dose.

It is interesting to note that the corrections anticipated by the RAYTRACE calculations which introduced a tapering of the U-sweeper poles and the use of the dynamic quadrupole $\mathrm{Q}_{\mathrm{C}}$, seem to be quite important for obtaining a round almost invariant spot shape independently of scanning, as shown in the measured spot patterns of fig. 15 . This was not the case, when we performed our first scans without quadrupole corrector connected. We finally decided to connect the quadrupole corrector in series with the U-sweeper. We conclude that the quadrupole corrector is a necessary beam optical element for the Gantry 2. 


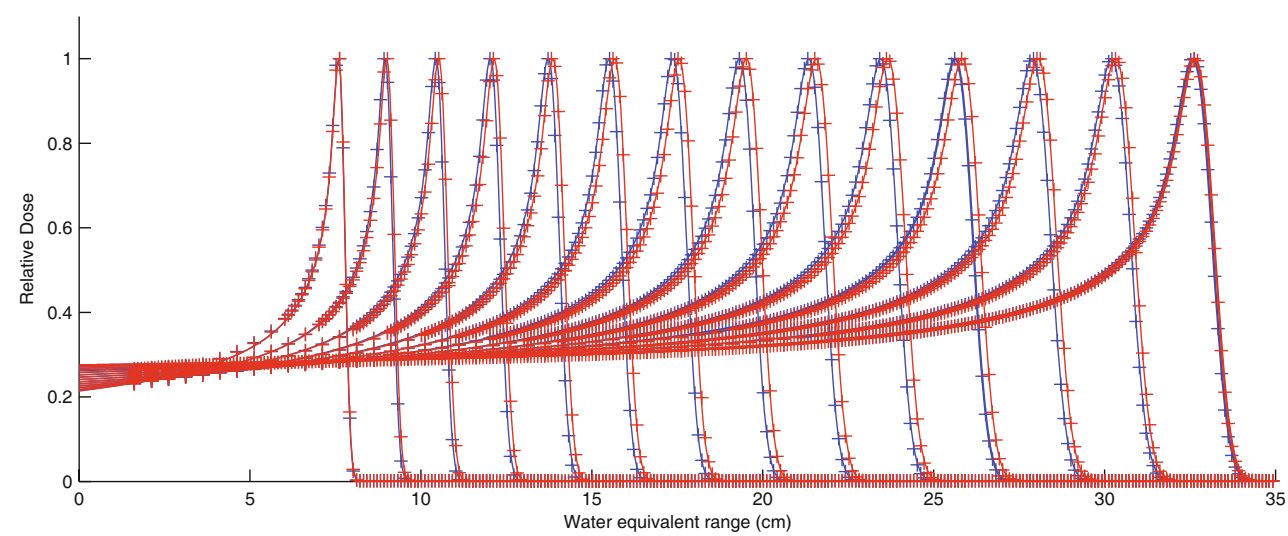

Fig. 16. Integral depth dose curves measured with a plane parallel $(8 \mathrm{~cm}$ diameter $)$ ionization chamber moved in depth in a water phantom. The curves are relative (normalized at the Bragg peak). The symbols + are measurements, the curves are dose model calculations to fit the data and to assign the nominal energy of the beam. We applied a regular magnet ramping from 100 to $230 \mathrm{MeV}$. In this experiment we observe hysteresis effects of the beam line. The data in red belong to the up-down scan, the data in blue to the down-up scan. We found hysteresis errors in the range of $1 \mathrm{~mm}$, which are corrected in the energy definition of the final tunes.

In order to achieve a round spot at the iso-center it was necessary to correct the setting of the first quadrupole $\mathrm{Q}_{1}$ of the gantry by about $10 \%$ for all energies. We could not find a reason for this difference, which is the only significant observed discrepancy to the settings derived from the calculations.

\subsection{Realization of a scanning system with fast energy changes}

\subsubsection{Beam tuning with cyclic ramping}

During delivery of the scanned dose field the beam line is set to a new energy by the beam delivery control system, which sends the data of all beam line elements in a single command to the machine control system. These data are pre-calculated and stored in the individual steering file(s) of each patient. The machine control system sets the beam line according to the received tune data and sends a ready signal to resume beam delivery, when the change of the energy has been completed. In this way the user in the gantry area has complete control over the whole beam line from the accelerator to the gantry. Based on the experience with Gantry 1 we rely on the long term reproducibility of the energy tunes, which are set without any retuning of the beam.

For Gantry 2 we decided to develop tunes by scanning the beam energy up-down and down-up following a cyclic loop between $230 \mathrm{MeV}$ and $70 \mathrm{MeV}$.

We found systematic effects in the measured proton ranges in water caused by magnetic hysteresis effects of the beam line. When we tune the beam line with the same current values in the power supplies with a given ramp up-down or down-up cycle we observed changes in the proton range. These systematic errors were of the order of $\pm 1 \mathrm{~mm}$. We correct for these effects by producing two different sets of tunes, one for the up-down and one for the down-up cycle. The beam line can be set to any continuous energy value in the range between 70 and $230 \mathrm{MeV}$. The nominal beam energy is defined in the first place by the measured proton range in water.

Figure 16 shows hysteresis effects of the up-down and down-up cycle (prior to introducing the hysteresis corrections). The depth dose curves with the Bragg peaks were measured with a large parallel ionization chamber moved in depth in a water phantom. The symbols are the measurements. The lines are fits of the measured curves using the dose model described in [14].

Figure 17 shows the same kind of data measured with a stack of ionization chambers (a multi-layer ionization chamber MLIC developed at PSI). The blue curves are dose-range data obtained when using a proper sequence of the tunes with respect to the ramp direction. The red curves are the same results, when the ramping sequence is intentionally mistaken. If the tuning sequence is correctly adhered to, the hysteresis errors are reduced from $1 \mathrm{~mm}$ down to a few tens of a mm.

\subsubsection{A gantry for very fast energy changes}

The beam line has been realized to the goal of applying changes of the beam energy as quickly as possible.

We were pleased to see that we can apply energy changes within about $80 \mathrm{~ms}$ for changes of the proton range in water of about $5 \mathrm{~mm}$. This is an order of magnitude faster than any other system so far. 


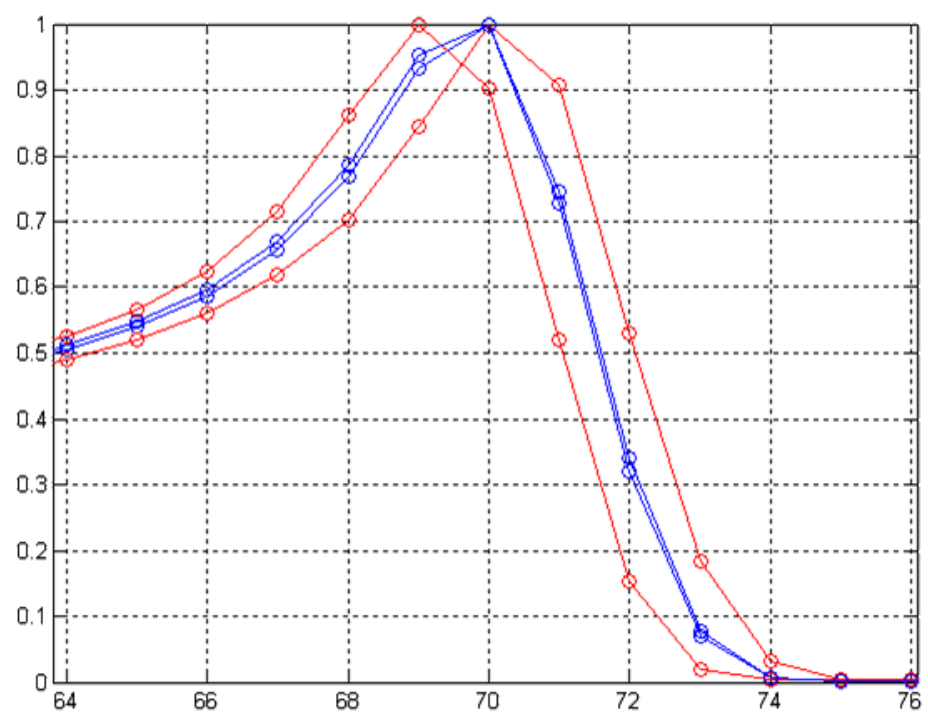

Fig. 17. Verification of the beam range in water measured with in a stack of $12810 \times 10 \mathrm{~cm}$ broad ionization chambers (the symbols are depth points spaced by about $1 \mathrm{~mm}$ thick aluminum plates, corresponding to roughly $2 \mathrm{~mm}$ steps in water). We check here the energy of a tune within an hysteresis loop of $100-230 \mathrm{MeV}$. The blue curves shows the proton range if the ramps up/down and down/up are performed in the correct order (errors are in the order of tens of mm). The red curves are if the up/down down/up ramps are applied in the wrong order. The error is then in the order of $1 \mathrm{~mm}$.

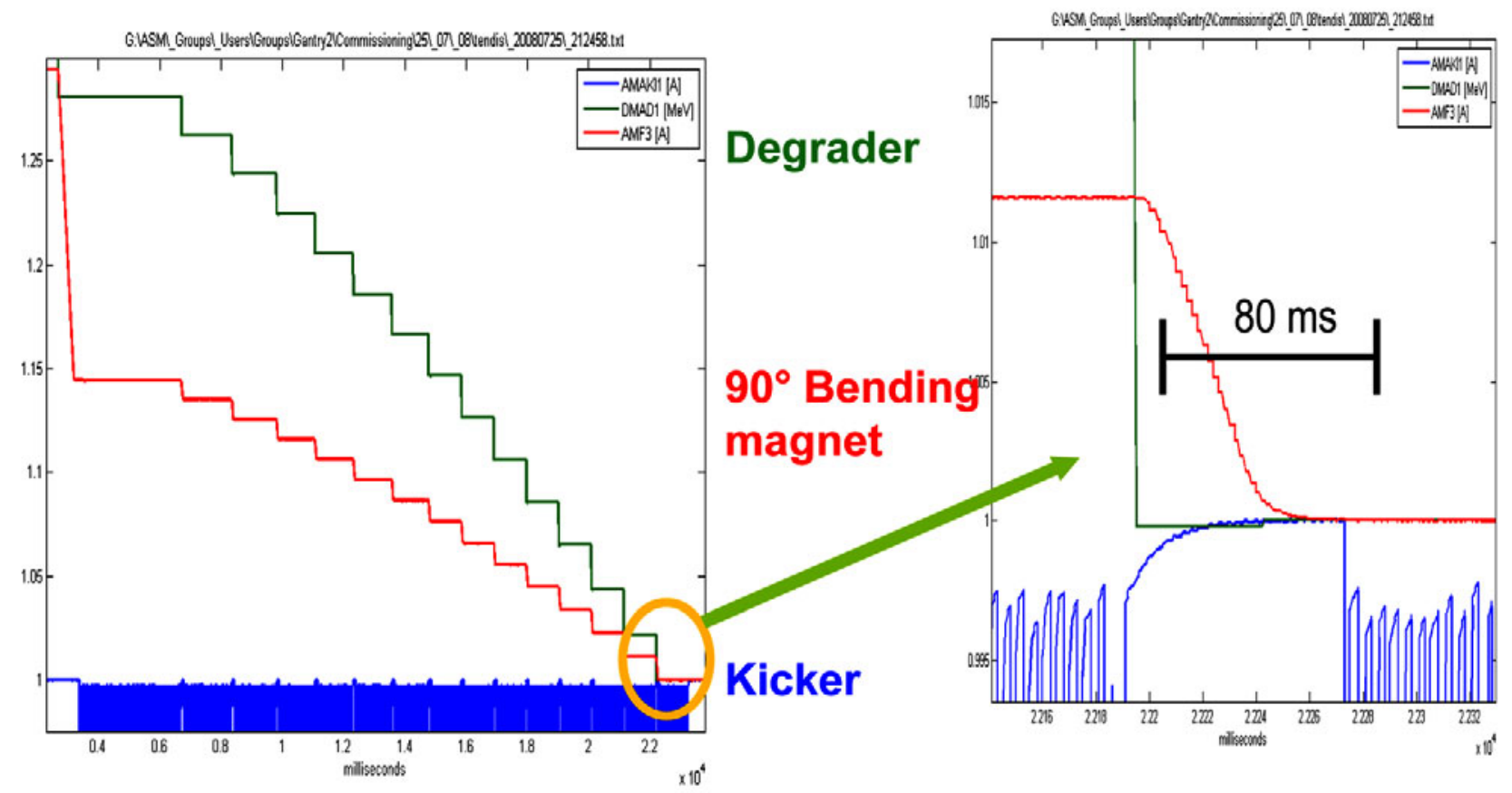

Fig. 18. Recorded values of the settings of the degrader (nominal value in green), last bending magnet (magnet current in red) and kicker (magnet field in blue, showing the beam on off of the spots) during the delivery of a box scan. In the right picture we expanded the image at one energy step, which shows that the change of the energy is performed within $80 \mathrm{~ms}$ (the value limits are given by the last magnet).

Figure 18 shows some time graphs taken during delivery of a scan field for a dose box. The beam on-off of the beam delivery of the spots is recorded as the blue line. In green we see the changes in the setting of the degrader. The line in red is the current in the last bending magnet, which is slightly slower in speed than the degrader. From this graph we highlighted the region in the last energy change showing that the transition in energy is within about $80 \mathrm{~ms}$. 


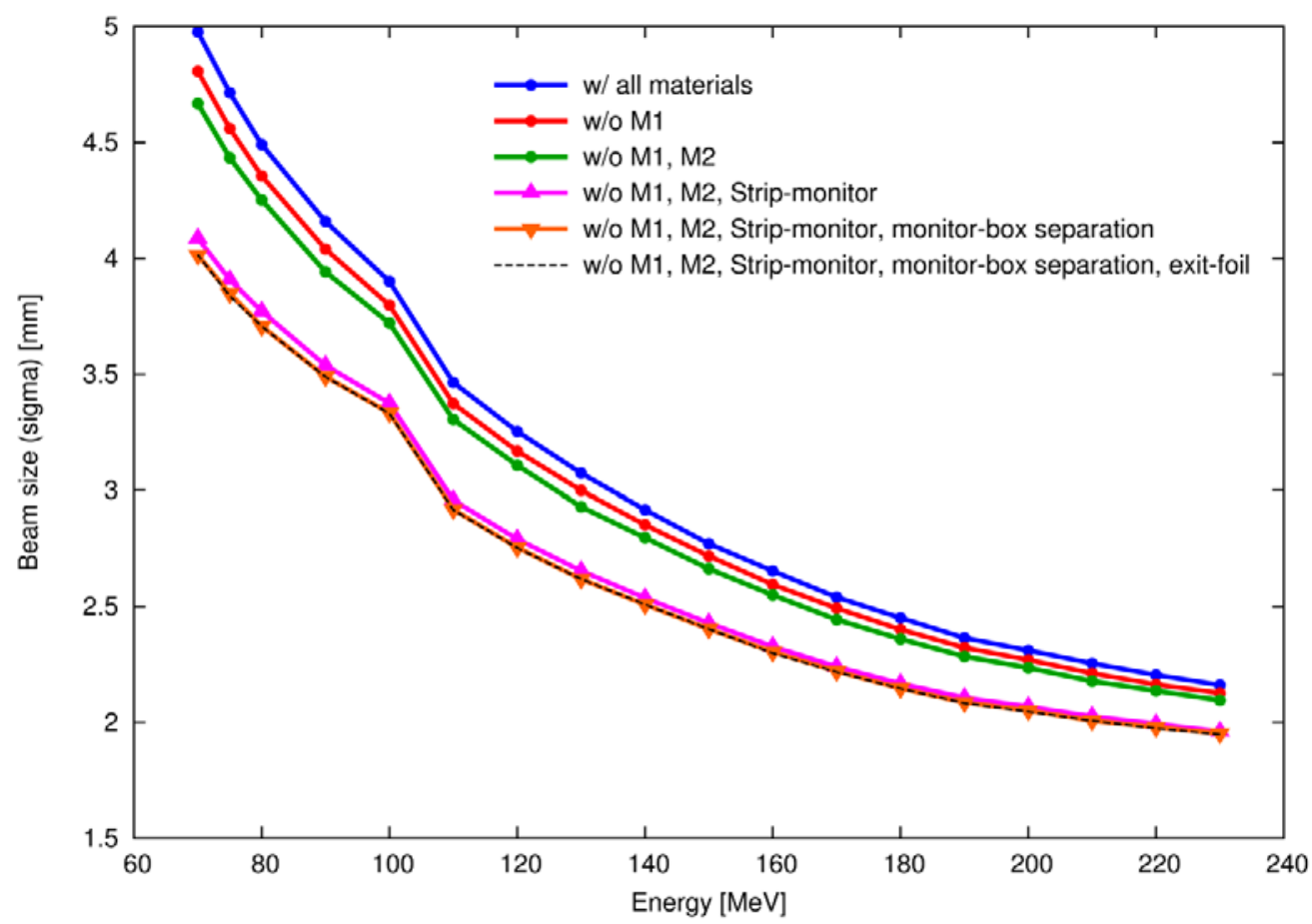

Fig. 19. Measured beam size sigma (FWHM/2.355) at the iso-center as a function of the beam energy in the $\mathrm{T}$ direction. The curves show the effect of removing in sequence the transmission monitors, the strip-monitor, the sheet that separate the monitor-box and the foil of the exit of the nozzle.

The use of fast energy changes is desired for being able to apply scanning with volumetric repainting, a scanning strategy which in our opinion should render scanning sufficiently robust against organ motion in most clinical situations.

We have however observed systematic drifts of the position of the beam at the iso-center of the order of $0.4 \mathrm{~mm}$ after small energy changes, i.e. those which are typical for energy steps within the spread out Bragg peak (SOBP). Larger beam position displacements in the range of $1-3 \mathrm{~mm}$ are observed when we change the energy with very big energy steps (of the order of the full energy range). All dynamic beam displacements behave like an exponential decay in time with a time constant of a couple of seconds. We plan to use a predictive model of these beam position displacements, which provides on-line correction to the U-sweeper as a function of the time after the last applied energy change. An alternative option would be to use the data from the strip monitor for a feed-back position correction loop. Another approach would be to ramp the magnets "long" before starting the scan and repaint only the SOBP without going through the whole energy cycle (if we can accept related errors in range $<1 \mathrm{~mm}$ ).

All this is work in progress. More experience has to be gained with the real system but from the first results we are confident that we will find a very good solution for applying the beam with high positional precision and with fast energy changes.

\subsection{Measured spot size}

The gantry nozzle has been designed with a minimal amount of material in the beam in order to keep small the broadening of the proton pencil beam due to scattering. Another strategy has been to place the thicker elements of the nozzle near the nozzle exit at the shortest distance to the patient.

With these precautions we achieved having a sharp pencil beam with a beam width $\sigma$ which is still less than 5 mm even at the lowest beam energies of $70 \mathrm{MeV}$.

Figure 19 shows the measured beam size at the iso-center as a function of the beam energy.

In this figure we see the effect on the beam size of adding one by one each element of the nozzle. We see that the amount of material in the nozzle is not contributing much to the size of the beam in air. A beam size of about $3 \mathrm{~mm}$ standard deviation $\sigma$ is in our opinion a good compromise. This value is at most energies smaller than the (inevitable) broadening effect of multiple scattering within the patient, which is affecting mostly the beam size at the Bragg peak depth. At the lowest energies we see that the spot is dominated by the phase space of the beam in air, but we see also that the scattering due to the material of the nozzle starts to become significant.

We are convinced that the data of Gantry 2 compare favorably with other scanning systems. 


\subsection{Status of the mechanical systems}

The commissioning of the mechanical systems is still ongoing.

For the moment we can report only with regard to the mechanical precision of the iso-center. We were pleased to see that the mechanical iso-centre of Gantry 2 is stable within a radius $\pm 0.35 \mathrm{~mm}$. The data were obtained with a distance sensor mounted on an arm attached to the nozzle by measuring in the $\mathrm{U}, \mathrm{T}$ and $\mathrm{S}$ direction the distance to a fixed cylinder mounted on the patient table while rotating the gantry. The center of gravity of these distance points defines the position of the mechanical iso-center.

The parts which are still missing and are under development, are the remote control of the motion of the patient table and of the gantry and the precise definition of the coordinates of the patient table including the bending corrections of the table due to the varying weights put on the table. The bending errors will be corrected depending on the applied load and torque measured with sensors mounted on the patient table. The implementation of results of the survey engineering into the steering software of the mechanical systems is another issue to be done.

\subsection{Start of the exploration of new advanced pencil beam scanning methods}

The beam delivery system of Gantry 2 has been conceived for providing fast scanning with volumetric repainting for treating moving targets.

The first use of the system will be based on discrete spot scanning (sequence of static spots delivered with beam switched off when moving from spot to spot), following the well established methods used since a decade with Gantry 1. The major difference of Gantry 2 compared to Gantry 1 will be the use of a double parallel magnetic scanning and the use of the fast energy changes ( $80 \mathrm{~ms}$ per SOBP step) realized within the beam line and the degrader (instead of a single magnetic scan axis and the use of the range shifter in front of the patient used on Gantry 1). We plan to apply discrete spot scanning with volumetric repainting by using the so-called iso-layer repainting method described in [21]. With this mode we expect to be able to treat with scanning moderately moving targets with motions below $\sim 5$ mm.

The control system has been designed to cope with a more futuristic beam delivery approach, which is to paint the dose with a continuous motion of the pencil beam, along lines, meanders or even contours. The control of the dose painting within an energy layer is based on the use of field-programmable gate arrays (FPGA), which allow defining arbitrary beam motion patterns (within an energy layer) by using tabulated data of the U- and T-sweeper position including variations of the beam intensity I controlled via the deflector plate. We have decided to explore first the approach to deliver the dose of layers programmed as a function of the real time. For this we are installing a feed-back loop, which adjusts dynamically the beam intensity measured with the gantry beam monitors to correspond to the dose rate values described in the intensity I-table of the FPGA. With this system we can program the motion of the sweepers with variable patterns and velocities (with maximum velocity of $2 \mathrm{~cm} / \mathrm{ms}$ in the $\mathrm{T}$ and $0.5 \mathrm{~cm} / \mathrm{ms}$ in the $\mathrm{U}$ direction). Along these beam patterns we can change the intensity of the beam with the deflector plate with a response time of about $100-200 \mu \mathrm{s}$.

The choice to work as a function of the real time is based on the idea to have a more deterministic beam delivery system, where corrections of systematic effects could be easier to control.

Figure 20 shows as an a example two dose images delivered and recorded with a scintillator screen seen by a CCD camera over a $45^{\circ}$ mirror. The scan of a whole dose image (of an energy layer) is programmed in the FPGA with the corresponding tables. We paint 11 lines of $10 \mathrm{~cm}$ length with $100 \%$ dose in the first and the last $5 \mathrm{~mm}$ portions of each line. In the central part of the line we change the beam intensity in steps of $10 \%$ from line to line. The U motion of the beam from one line to the next adjacent line is masked by programming the beam intensity of the U motion segments with zero dose.

In the first picture 20a the lines were painted by changing the velocity of the beam using the sweepers. The deflector plate was used there only to switch the beam off during the stepping in U. The dose layer was painted only once within a time of $760 \mathrm{~ms}$.

In fig. $20 \mathrm{~b}$ we explored the possibility to scan the lines in $\mathrm{T}$ with maximum velocity (with $5 \mathrm{~ms}$ per line) while adapting the dose to the prescribed value by modulating the intensity of the beam with the deflector plate. In order to obtain an image with a similar exposure as in 20a we have repainted the image 23 times. The result looks very similar and is indeed very encouraging.

The deflector plate is the fastest beam delivery device available on Gantry 2 for dose painting. We thus expect that the exploration of this device is of relevance for achieving maximum repainting capability.

These preliminary results underline our intention to develop a scanning system with high repainting numbers applied to layers and volumes.

The idea is to apply energy layers within typically $200 \mathrm{~ms}$, with a dead time for the energy changes of less than $100 \mathrm{~ms}$. A total volume scan time of $6 \mathrm{~s}$ per liter could be possible if we consider only maximum scanning speed, not including possible limitations due to the maximum available instantaneous dose rate (and required total dose). The long-term goal is to apply about 10 volumetric repainting for a dose of $1 \mathrm{~Gy}$ in a volume of 1 liter in a couple of 


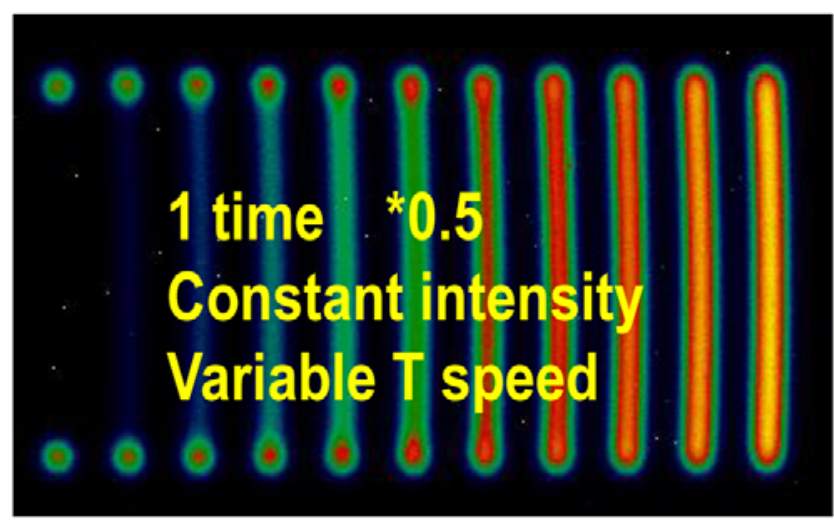

a)

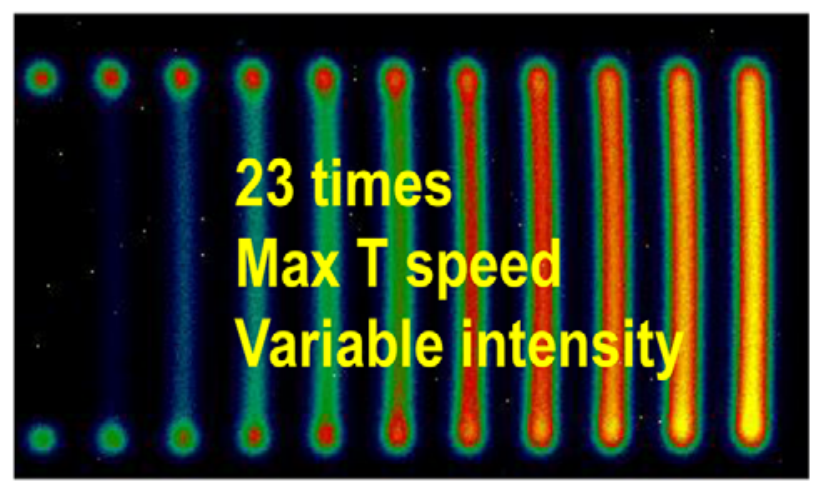

b)

Fig. 20. Painting of parallel dose lines programmed as T-U-I tables in the FPGAs. The upper picture is obtained by keeping the intensity I constant and by changing the velocity of the T-U sweepers. In the lower picture the vertical lines are painted with maximum $\mathrm{T}$ velocity $(10 \mathrm{~cm}$ in $5 \mathrm{~ms})$. The shaping of the dose is obtained by changing the intensity I of the beam. The lower image was applied 23 times.

minutes. A recent study published by our group [21] has shown that this approach is very promising in the context of robustness against organ motion.

Recently we could show that with Gantry 2 it is principally possible to deliver 0.1 Gy conformally to a sphere of half a liter within $7 \mathrm{~s}$ using the continuous line scanning method described above. This approach could be used for treating the whole target of a small lung tumor within a single breath-hold, which would be then repeated as a sequence of static irradiations to provide repainting. This could be an interesting alternative to gating which is another new issue to be explored with scanning.

The feasibility of uniform scanning on the PSI Gantry 2 as a method for simulating scattering on a scanning gantry has been successfully shown experimentally, as reported in a recent publication [22].

\section{Conclusions}

The basic installations for the new Gantry 2 of PSI have been recently completed. The initial commissioning results look very promising. The system contains many new features of potential interest for further advancing the technology of pencil beam scanning.

We encountered some unexpected difficulties in the realization of the gantry system, mainly with the fabrication of the last $90^{\circ}$ bending magnet. All problems could be solved in a satisfactory way. Some of the problematic effects could be improved by changing slightly the design of the last bending magnet in a next prototype.

We are confident that the new system will be competitive in view also of the new innovative gantry layout. Gantry 2 offers maximum flexibility in choosing beam incidence while preserving an easy permanent access to the patient table at the iso-center. The new devices for image-guidance - the BEV X-ray equipment and the in-room sliding $\mathrm{CT}$ - should pave the way to develop image guidance in proton therapy, which is a new very important topic in the context of treating moving targets with proton beams.

The beam optics calculations proved to be correct. Tolerable differences between the experimental results and the design were found in connection with the non-linear behavior of the magnetic field of the last $90^{\circ}$ bending 
magnet. The non-linearities of the sweeper maps are bigger than expected and show a strong dependence with beam energy.

Solutions to these problems were found by providing detailed specific maps of the action of the sweeper magnets on the position of the scanned beam, including reproducible hysteresis effects on beam position and range due to partial saturation of the dipoles in the beam line.

We could show that Gantry 2 delivers the dose with a small pencil beam at all energies, even at very low energy. This is important for the quality of the dose distribution in terms of sharpness of the lateral fall-off.

We could prove that the system is capable to scan the energy along a SOBP in small range steps not taking more than $80 \mathrm{~ms}$ per step.

The sweeper magnets provide fast continuous scanning at high velocity, especially in the non-dispersive direction of the scan.

Gantry 2 delivers conformal dose fields in a fully automated way.

Beam losses in the degrader system as a function of the beam energy, producing beam intensity variations over two orders of magnitudes could be compensated by producing intentional losses at selected collimator holes. We thus found in a restricted energy window a set of intensity-compensated energy tunes, which allow exploring fast beam scanning with dynamic modulation of the beam intensity for dose painting.

A pre-absorber block will be used to close the gap to the energies below $100 \mathrm{MeV}$ when working with dose painting with beam intensity modulation, respectively, $70 \mathrm{MeV}$ when working with discrete spot scanning.

First tests on the principal feasibility of fast painting of dose lines with dose shaping by modulating the beam intensity at the ion source look very encouraging.

The system is therefore almost ready for the demonstration of conformal volumetric target repainting with continuous lines painting.

Concerning the future clinical use of the system we are optimistic about the performance of the new Gantry 2.

For treating static tumors we will use the Gantry 2 with discrete spot scanning with energies down to $70 \mathrm{MeV}$. We should thus be able to reach the highest possible precision, since we will be working with almost no material in the beam line. If necessary we will use at low energy optional collimators added on top of conformal scanning.

With continuous line painting with beam intensity modulation, the system can deliver quickly whole energy layers, within a time which is small compared to the respiration cycle. This will make the system more robust against organ motion. We have a system which in addition provides fast energy changes and which is thus well suited for applying volumetric repainting. We expect that the possibility to apply volumetric repainting instead of repainting only energy layers could be an advantage for treating moving targets.

If we will manage to deliver a conformal scanned dose on a small volume in less than $5-10 \mathrm{~s}$ with sufficient precision, we could envisage treating lung nodules using a breath-hold technique by applying the dose to the whole target volume within each single breath-hold cycle.

The main idea of the Gantry 2 is to provide new advanced scanning techniques such as to make pencil beam scanning a universal beam delivery method, capable of replacing scattering in most, if not all situations.

The premises to reach this goal seem from our preliminary experience realistic and within reach. The way which remains to go for demonstrating these ideas in practice with patient treatments is however still very long and paved with small but numerous troubles, the famous devil trapped in the precision details.

We hope that the new ideas embedded in Gantry 2 will have an impact on the future of proton therapy. By this alone the project would be justified.

We would like to thank the technical experts of PSI, in particular D. George, M. Negrazus, A. Gabard and V. Vrankovic for the realization of the beam line elements of Gantry 2 and for providing to us the measured magnetic data and together with U. Heidelberger and the vacuum group of PSI for providing a good solution to the vacuum problems of the last bending magnet. R. Künzi for the fine tuning of the critical power supplies. We thank R. Kobler for his excellent management for the installation work of the technical systems of Gantry 2. We thank S. Lin and S. König for providing the monitor systems. We thank M. Schippers for the help given in connection with the investigations with the deflector plate and for giving us access to a second beam defocusing alternative in the beam line region ahead of the degrader.

\section{References}

1. M. Phillips et al., Phys. Med. Biol. 37, 223 (1992).

2. C.V. Essen et al., Int. J. Radiat. Oncol. Biol. Phys. 8, 1499 (1982).

3. E. Hug et al., PSI Scientific Report 2009: http://www.psi.ch/info/MediaBoard/PSI_ScientificReport_2009.pdf.

4. E. Pedroni et al., Med. Phys. 22, 37 (1994).

5. T. Haberer et al., Nucl. Instrum. Methods A 330, 296 (1993).

6. A.J. Lomax et al., Phys. Med. Biol. 44, 185 (1999). 
7. E. Pedroni, in Treatment Planning for External Beam Therapy with Neutrons, edited by G. Burger, J.J. Broerse (Urban \& Schwarzenberg, Munich, 1981) pp. 60-69.

8. E. Pedroni, Radiat. Environm. Biophys. 16, 211 (1979).

9. E. Pedroni, in Proton and Charged Particle Radiotherapy, edited by T.F. DeLaney, H.M. Kooy (Williams and Wilkins, Boston, MA, 2008) chapt. 5b.

10. J.M. Schippers, Rev. Acc. Science and Technology 2, 179 (2009).

11. E. Pedroni et al., Z. Med. Phys. 14, 25 (2004).

12. A. Gabard et al., Proceedings of the 21st International Conference on Magnet Technology, MT-21, IEEE Trans. Appl. Supercond. 20, 794 (2010).

13. K.L. Brown et al., yellow reports CERN 73-16 (1973) \& CERN 80-04 (1980).

14. E. Pedroni et al., Phys. Med. Biol. 50, 541 (2005).

15. Calculation using the Graphic TRANSPORT Framework of U. Rohrer at PSI: http://aea.web.psi.ch/Urs_Rohrer/MyWeb/ trans.htm.

16. S. Kowalski, H.A. Enge, RAYTRACE, MIT, Cambridge MA USA, 1987.

17. E. Pedroni, H. Enge, Med. \& Biol. Eng. \& Comput. 33, 271 (1995).

18. D.C. Carey, K.L. Brown, F. Rothacker, Fermilab-Pub-95/0: http://lss.fnal.gov/archive/1998/pub/Pub-98-310.pdf.

19. J.F. Ziegler, SRIM and TRIM, http://www.srim.org/.

20. S.N. Boon et al., Med. Phys. 27, 2198 (2000).

21. S.M. Zenklusen, E. Pedroni, D. Meer, Phys. Med. Biol. 50, 5103 (2010).

22. S.M. Zenklusen et al., to be published in Med. Phys. in 2011. 